\title{
Mediators in position auctions
}

\author{
Itai Ashlagi ${ }^{a}$, Dov Monderer ${ }^{\mathrm{b}, *}$, Moshe Tennenholtz ${ }^{\mathrm{b}, \mathrm{c}}$ \\ ${ }^{a}$ Harvard Business School, Harvard University, 02163, USA \\ b Faculty of Industrial Engineering and Management, Technion-Israel Institute of Technology, Haifa 32000, Israel \\ c Microsoft Israel REDD Center, 13 Shenkar St., Herzeliya 46725, Israel
}

\section{A R T I C L E I $\mathrm{N}$ F O}

\section{Article history:}

Received 25 July 2007

Available online 28 November 2008

\section{JEL classification:}

D44

C72

Keywords:

Mediator

Position auction

Implementation

\begin{abstract}
A B S T R A C T
A mediator is a reliable entity which plays on behalf of the players who give her the right to play. The mediator acts in a pre-specified way based on messages received from the players. However, a mediator cannot enforce behavior; that is, players can play in the game directly without the mediator's help. A mediator generates a new game for the players, the mediated game. The outcome in the original game of an equilibrium in the mediated game is called a mediated equilibrium. Monderer and Tennenholtz introduced a theory of mediators for games with complete information. We extend the theory of mediators to games with incomplete information, and apply the new theory to position auctions, a central topic in electronic commerce. We provide a minimal set of conditions on position auctions, which is sufficient to guarantee that the VCG outcome function is a mediated equilibrium in these auctions.
\end{abstract}

(c) 2008 Elsevier Inc. All rights reserved.

\section{Introduction}

In a game with incomplete information with private values every player holds some private information, which is called the player's type, and has a set of possible actions. Every profile of the players' actions generates an outcome in a given set of possible outcomes. The utility of a player depends on the vector of types and on the outcome generated by the profile of actions. A strategy of a player is a function that maps each of its possible types to an action. The game is called a Bayesian game when there exists a commonly known probability measure over the profiles of types. Otherwise, it is called a preBayesian game. In this paper we deal only with pre-Bayesian games. The leading solution concept for pre-Bayesian games is ex post equilibrium: a profile of strategies, one for each player, such that no player has a unilateral profitable deviation from it, independent of the types of the other players. Consider the following simple example of a pre-Bayesian game, which possesses an ex post equilibrium. The game is denoted by $\mathbb{H}$, and the set of outcomes in this game is identified with the set of profiles of actions.

\begin{tabular}{c|c|c|} 
& \multicolumn{1}{c}{$a$} & $b$ \\
\cline { 2 - 3 }$a$ & 5,2 & 3,0 \\
\cline { 2 - 3 }$b$ & \multicolumn{1}{c}{0,0} & 4,2 \\
\cline { 2 - 3 } & \multicolumn{2}{c}{$\mathrm{A}$}
\end{tabular}

\begin{tabular}{c|c|c|}
\multicolumn{1}{c}{} & \multicolumn{1}{c}{$a$} & \multicolumn{1}{c}{$b$} \\
\cline { 2 - 3 }$a$ & 2,2 & 0,0 \\
\cline { 2 - 3 }$b$ & $2,3,3$ & 5,2 \\
\cline { 2 - 3 } & \multicolumn{3}{c}{3}
\end{tabular}

\footnotetext{
An early version of this paper appears in the proceedings of the ACM conference on Electronic Commerce 2007 (EC'07). The current version significantly extends the conference version; it introduces a general model of mediators in games with incomplete information, includes all proofs, and includes further discussion and exposition. This work has been partially supported by the Israel Science Foundations (ISF).

* Corresponding author.

E-mail addresses: iashlagi@hbs.edu (I. Ashlagi), dov@ie.technion.ac.il (D. Monderer), moshet@ie.technion.ac.il (M. Tennenholtz).
} 
In $\mathbb{H}$ there are two players. Each player can choose one out of the two actions, $a, b$. The column player, player 2 , has a private type, $A$ or $B$. The row player, player 1 , has only one possible type. A strategy of player 1 is $g_{1}$, where $g_{1}=a$ or $g_{1}=b$. A strategy of player 2 is a function $g_{2}:\{A, B\} \rightarrow\{a, b\}$. That is, player 2 has 4 possible strategies. In this game the strategy profile $\left(g_{1}, g_{2}\right)$ is an ex post equilibrium, where $g_{1}=b$ and $g_{2}(A)=b, g_{2}(B)=a$. Unfortunately, pre-Bayesian games do not, in general, possess ex post equilibria, even if we allow mixed strategies. In order to address this problem and enable the players to reach a desired outcome of a given game as an ex post equilibrium we suggest in this paper the use of mediators. A mediator for a given game is a reliable entity that can interact with the players and perform actions on their behalf. However, a mediator cannot enforce behavior. Indeed, a player is free to participate in the game without the help of the mediator. The mediator's behavior on behalf of the players that give it the right of play is pre-specified, and is conditioned on information the players provide to the mediator. This notion is natural; in many systems there is some form of reliable party or administrator that can be used as a mediator. Brokers and routers are simple examples of such mediators. Notice that we assume that the game is given, and all the mediator can do is to perform actions on behalf of the players that explicitly allow it to do so. ${ }^{1}$ In order to illustrate the power ${ }^{2}$ of mediators for games with incomplete information consider the following pre-Bayesian game $\mathbb{G}$ that does not possess an ex post equilibrium. In $\mathbb{G}$, the column player has two possible types: $A$ and $B$.

\begin{tabular}{l|c|c|}
\multicolumn{1}{c}{} & \multicolumn{1}{c}{$a$} & $b$ \\
\cline { 2 - 3 }$a$ & 5,2 & 3,0 \\
\cline { 2 - 3 }$b$ & \multicolumn{1}{c}{0,0} & 2,2 \\
\cline { 2 - 3 } & \multicolumn{2}{c}{$\mathrm{A}$}
\end{tabular}

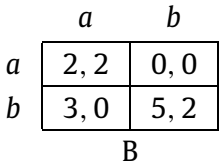

A mediator for $\mathbb{G}$ should specify the actions it will choose on behalf of the players that give it the right to play. If player 2 wants to give the mediator the right to play it should also report a type. Consider the following mediator: If both players give the mediator the right to play, the mediator will play on their behalf $(a, a)$ if player 2 reports $A$, and $(b, b)$ if player 2 reports $B$. If only player 1 gives the mediator the right to play, the mediator will choose $a$ on his behalf. If only player 2 gives the mediator the right to play, the mediator will choose action $a$ (resp. $b$ ) on his behalf, if $B$ (resp. $A$ ) has been reported. The mediator generates a new pre-Bayesian game, which is called the mediated game. In the mediated game player 1 has three actions: give the mediator the right to play, denoted by $m$, or play directly $a$ or $b$. Player 2 has four actions: $m-A$, $m-B, a, b$, where $m-A(m-B)$ means reporting $A(B)$ to the mediator and giving it the right to play. The mediated game is described in the following figure:

\begin{tabular}{|c|c|c|c|c|}
\hline & $m-A$ & $m-B$ & $a$ & $b$ \\
\hline$m$ & 5,2 & 2,2 & 5,2 & 3,0 \\
\hline$a$ & 3,0 & 5,2 & 5,2 & 3,0 \\
\hline$b$ & 2,2 & 0,0 & 0,0 & 2,2 \\
\hline \multicolumn{5}{|c|}{ A } \\
\hline \multirow[b]{2}{*}{$m$} & $m-A$ & $m-B$ & $a$ & $b$ \\
\hline & 2,2 & 5,2 & 2,2 & 0,0 \\
\hline$a$ & 0,0 & 2,2 & 2,2 & 0,0 \\
\hline$b$ & 5,2 & 3,0 & 3,0 & 5,2 \\
\hline
\end{tabular}

It is easy to verify that giving the mediator the right to play, and reporting truthfully, is an ex post equilibrium at the mediated game. That is, $\left(f_{1}, f_{2}\right)$ is an ex post equilibrium, where $f_{1}=m$, and $f_{2}(A)=m-A, f_{2}(B)=m-B$. In this case we say that the mediator implements the outcome function $\varphi^{m}:\{A, B\} \rightarrow \mathbf{O}$ defined by $\varphi^{m}(A)=(a, a)$ and $\varphi^{m}(B)=(b, b)$, where $\mathbf{O}$ is the set of outcomes of $\mathbb{G}$.

\footnotetext{
1 This natural setting is different from the one discussed in the classical theories of implementation and mechanism design, where a designer designs a new game from scratch in order to yield some desired behavior.

2 Although we do not formally discuss "folk theorems" in this paper, we want to stress that an appropriate folk theorem could have explained some of the power of mediators. A folk theorem would have stated that for every equilibrium in the repeated game there exists a mediator that implements in the mediated game the outcome generated by this equilibrium. It is well known that the set of equilibrium outcomes of a repeated game is very rich, and therefore by a careful design of the mediator, the mediated game can lead to a desired outcome.
} 
The aim of this paper is twofold. We introduce mediators for games with incomplete information, ${ }^{3}$ and apply them in the context of position auctions. ${ }^{4}$

Our choice of position auctions as the domain of application is not a coincidence; indeed, position auctions have become a central issue in advertisement and sponsored search, and the selection of appropriate position auctions for that task is a subject of considerable amount of study (Mehta et al., 2007; Feng et al., 2006; Lahaie, 2006; Borgs et al., 2007; Edelman et al., 2007; Varian, 2007). There exist many types of 3rd parties (i.e. neither advertisers, nor the companies running the auctions) which do the bidding on behalf of advertisers in position auctions. ${ }^{5}$ The typical role of such a 3rd party is to decide on the exact keywords for which a bid will be made and on these bids' values. As thousands of position auctions are being held by the leading search engines and many advertisers seek the support of such 3rd parties, the market of 3rd parties for bidding in position auctions is flowering. The type of 3rd parties discussed in this paper allows for coordination of advertisers' bids in position auctions. ${ }^{6}$ The goal of a mediator is to maximize its own profit. This goal is not explicitly modeled in this paper. However, it is implicitly assumed that maximizing the mediator's profit is highly correlated with the goal of guaranteeing high utility to rational players. In this paper we deal with the implementation of the outcome of the VCG position auction by mediation. This is partially motivated by the findings of Varian (2007). Varian considers the next-price position auction, ${ }^{7}$ which is currently used in practice, and a natural subset of its equilibria under complete information. He shows that, within this subset, the utility of each player is maximized in the equilibrium which yields the VCG utilities. Furthermore, using empirical data, Varian claims that actual players' bids belong to the equilibria subset, but are not necessarily in the equilibrium that provides the maximal utility for the players. Hence, a mediator that implements the VCG outcome function will improve the players' utilities. Furthermore, by Ashlagi (2007), the VCG outcome function is the only anonymous function that can be truthfully implemented.

Notice that in our work we consider the existence of only a single mediator, while in some keyword markets there may be more than one possible mediator. However, there are keyword markets where there exists only a single mediator. Moreover, in markets where no mediator is active, our work provides a new form of mediation, which can lead the players to higher utilities. Finally, as observed in practice, search engines also provide mediation services. While increasing players' payoffs might seem conflicting to the search engine short term revenue, it is believed to lead to high revenue in the longer term, due to the increase in advertisers' satisfaction. Naturally, the role of the mediator could have been avoided by changing the rules of the underlying auction; however, such a change is considered problematic due to the need to modify the existing standards. One type of mediator for position auctions has already been discussed, for other purposes, in the literature: An English auction type of algorithm was constructed in Edelman et al. (2007); this algorithm takes as input the valuations of the players and outputs bids for the next-price position auction. It was proved there that reporting the true type to this algorithm by each player forms an ex post equilibrium, which generates the VCG outcome. In our language, this algorithm can almost be considered as a mediator for the next-price position auction that implements the VCG outcome function; What is missing, is a component that "punishes" players who send their bids directly to the auctioneer, and a proof that using the mediator's service and reporting the true type by each player is an ex post equilibrium in the mediated game defined by the algorithm and by the additional component. Notice that, in principle, a mediator may generate a desired outcome function by punishing the players who do not use its services using very high bids by the players that use its services. However, we believe that such mediators are not realistic, and therefore we concentrate on the search for individually rational mediators that implement the VCG outcome function and satisfy an additional rationality condition: The utility of a player who gives the mediator the right to play and reports his type truthfully cannot be negative regardless of the actions taken by the players who did not choose the mediator's services, or players who report false types to the mediator. We first prove the existence of such desired mediators for next-price position auctions. Next, we provide a characterization for the existence of individually rational mediators that implement the VCG outcome function in position auctions; we provide three conditions on position auctions that imply the existence of an individually rational mediator for any given position auction satisfying these conditions. We also show that the set of those three conditions is minimal. Using this result, we prove the existence of individually rational mediators for a rich class of position auctions, including all $k$-price position auctions, $k>1$. For $k=1$, the self-price position auction, we show an impossibility result. However, in

\footnotetext{
3 Mediators that can obtain the "right to play" but cannot enforce the use of their services have been defined and discussed for general $n$-person games with complete information in Monderer and Tennenholtz (2006); for games with complete information the main interest is in leading players to behaviors which are stable against deviations by coalitions (i.e. in strong equilibrium). A special case of mediators was already discussed in Kalai and Rosenthal (1976), where the authors discussed mediators for a two-person game, which is known to the players but not to the mediators, and they looked for Nash equilibrium in the new game generated by the mediator. The topic of mediators for games with complete information has been further generalized and analyzed in Rozenfeld and Tennenholtz (2007). A recent paper (Tauman-Kalai et al., 2007) implicitly discusses mediators in games with complete information via the notion of commitment-a commitment device serves as a mediator; this paper deals only with equilibrium (in contrast to strong equilibrium).

4 Mediators for Bayesian one-item auctions (in particular first price and second price auctions) have been already discussed in Graham and Marshall (1987), McAfee and McMillan (1992), Bhat et al. (2005). However, the mediators in these papers are endowed with an additional strong characteristic-the ability to re-distribute payoffs.

5 For example, http://www.efrontier.com and http://www.topclickmedia.co.uk.

6 Such coordination is known in economics, for example in the context of the so-called bidding rings. See the references mentioned in footnote 4.

7 In Edelman et al. (2007) next-price position auctions are called generalized second price (GSP) auctions.
} 
auctions with the first arrival rule, in which ties are impossible, we prove the existence of individually rational mediators that implement the VCG outcome function.

In Section 2 we present the general theory of mediators for pre-Bayesian games. In Sections 3-6 we apply mediators to position auctions. It Section 7 we generalize the results on position auctions to results on position auctions with quality factors, in which the auction organizer can express preferences over players. In such auctions we deal with the implementation of the outcome function of an appropriate weighted VCG position auction.

We end this introduction with a discussion of additional related literature. A weaker form of a mediator discussed in the game theory literature is captured by the notion of correlated equilibrium Aumann (1974). This notion was generalized to communication equilibrium in Forges (1986), Myerson (1986). An additional type of mediator is discussed in Monderer and Tennenholtz (2004a). However, in all these settings the mediator cannot perform actions on behalf of the players that allow it to do so. Another type of mediators is captured by the notion of conditional contracts. In a conditional contract, a joint strategy for the group of all players is suggested, and is executed only if all players agree to accept this suggestion. However, the idea that a mediation device will play also on behalf of a strict subset of the players, who give it the right to play, has not been considered in that literature.

\section{Mediators in pre-Bayesian games}

In this section we present a general theory of mediators in pre-Bayesian games. In a pre-Bayesian game, $\mathbb{G}$, there is a fixed set of players $N=\{1,2, \ldots, n\}$, each of the players is endowed with a set of actions $b_{i} \in B_{i}$. There is a set of outcomes $\mathbf{0}$, and a function $\psi: \mathbf{B} \rightarrow \mathbf{O}$ that maps action profiles to outcomes, where $\mathbf{B}=B_{1} \times \cdots \times B_{n}$. There is a set of states $\omega \in \Omega$. The payoff of player $i, w_{i}(\omega, a)$ depends on the realized state $\omega \in \Omega$, and on the outcome $a \in \mathbf{0}$. However, the realized state is not known to the players. Every player $i$ receives a state-correlated type, $v_{i}=\tilde{v}_{i}(\omega) \in V_{i}$ on which he conditions his action. Let $\mathbf{V}=V_{1} \times \cdots \times V_{n}$. In this paper we deal with private value auctions. Therefore, as is common for such auctions, we assume that $\Omega=\mathbf{V}$. Hence, for every $\mathbf{v}=\left(v_{1}, \ldots, v_{n}\right) \in \mathbf{V}, \tilde{v}_{i}(v)=v_{i}$.

Let $\mathbb{G}=\left(N, \mathbf{V}, \mathbf{O},\left(w_{i}\right)_{i \in N}, \mathbf{B}, \psi\right)$ be a pre-Bayesian game. We define the utility function of $i, u_{i}: \mathbf{V} \times \mathbf{B} \rightarrow \mathbb{R}$ as follows:

$$
u_{i}(\mathbf{v}, \mathbf{b})=w_{i}(\mathbf{v}, \psi(\mathbf{b})) .
$$

A strategy of player $i$ in $\mathbb{G}$ is a function $f_{i}$ that assigns an action, $b_{i}=f_{i}\left(v_{i}\right) \in B_{i}$, to every possible type $v_{i} \in V_{i}$. A profile of strategies $\mathbf{f}=\left(f_{1}, \ldots, f_{n}\right)$ is an ex post equilibrium in $\mathbb{G}$ if for every player $i$, and for every $\mathbf{v} \in \mathbf{V}$

$$
u_{i}(\mathbf{v}, \mathbf{f}(\mathbf{v})) \geqslant u_{i}\left(\mathbf{v}, b_{i}, \mathbf{f}_{-i}\left(\mathbf{v}_{-i}\right)\right), \quad \forall b_{i} \in B_{i},
$$

where $\mathbf{f}(\mathbf{v})=\left(f_{1}\left(v_{1}\right), \ldots, f_{n}\left(v_{n}\right)\right)$, and $\mathbf{f}_{-i}\left(\mathbf{v}_{-i}\right)$ is the vector $\mathbf{f}(\mathbf{v})$ without the $i$ 's component. ${ }^{8} f_{i}$ is a dominant strategy for $i$ if the above inequalities hold for every profile $f_{-i}$ of the other players' strategies.

In mechanism design theory, there is a given environment, $\mathbb{E}=\left(N, T, \mathbf{0},\left(w_{i}\right)_{i \in N}\right)$ that contains all components of a preBayesian game except for the rules of the game; that is, the environment does not contain the action sets and the mapping from actions to outcomes. In addition, the mechanism designer has an outcome function $\varphi: \mathbf{V} \rightarrow \mathbf{0}$, which she wishes to implement. Hence, the goal of the designer is to find rules, $(\mathbf{B}, \psi)$ and an ex post equilibrium $\mathbf{f}$ in the pre-Bayesian game $\mathbb{G}=(\mathbb{E}, \mathbf{B}, \psi)$ that implements $\varphi$ in the sense that

$$
\psi(\mathbf{f}(\mathbf{v}))=\varphi(\mathbf{v}), \quad \forall \mathbf{v} \in \mathbf{V} .
$$

In contrast, in this paper we consider situations in which the pre-Bayesian game is given, and cannot be changed. It is well known that ex post equilibrium need not exist even in very simple pre-Bayesian games. This makes the choice of a strategy for a participant very difficult. To deal with the non-existence problem, and/or to increase players' utility when a particular ex post equilibrium is not desirable, and/or to guarantee economic efficiency, we suggest the use of mediators. A mediator is a reliable party that acts in a pre-specified way on behalf of the players who give her the right to do so. All other players can play independently or use other mediators. Mediators for games with complete information have been defined and analyzed in Monderer and Tennenholtz (2006). In games with complete information the only input the mediator collects is the "right to play." ${ }^{\prime 9}$ Hence, her action depends on the set of players that give her the right to play. Therefore, a mediator for a game with complete information is defined by a vector $\mathbf{b}=\left(\mathbf{b}_{S}\right)_{S \subseteq N}$, where $\mathbf{b}_{S}=\left(b_{i}\right)_{i \in S} \in \mathbf{B}_{S}$ is the vector of actions used by the mediator if $S$ is the set of players that give her the right to play. ${ }^{10}$ In a pre-Bayesian game $\mathbb{G}$, the mediator can use the information provided by the players. Hence, a player is required not only to give the mediator the right to play but also to report his type.

\footnotetext{
8 It is well known that an ex post equilibrium in a pre-Bayesian game is a Bayesian equilibrium for every choice of prior probability and vice versa. Indeed, the classical literature in economics/game theory actually discussed ex post equilibrium in a particular Bayesian game, and defined it as a Bayesian equilibrium which is robust to changes in the prior probability. Only recently the concept of pre-Bayesian games have been explicitly defined and analyzed. See e.g., Holzman and Monderer (2004), Holzman et al. (2004), where pre-Bayesian games are called games in informational form and games without probabilistic information, Hyafil and Boutilier (2004), where they are called games with incomplete information with strict type uncertainty, Aghassi and Bertsimas (2006), where they are called distribution-free games with incomplete information, and Ashlagi et al. (2006), where they are called pre-Bayesian games.

${ }^{9}$ What we call here a mediator for games with complete information is called a minimal mediator in Monderer and Tennenholtz (2006). It is proved in Monderer and Tennenholtz (2006) that a minimal mediator can implement every outcome which can be implemented by a mediator.

${ }^{10}$ In general, $\mathbf{b}_{S}$ can be a correlated strategy.
} 
Definition 1 (Mediators). Let $\mathbb{G}=(\mathbb{E}, \mathbf{B}, \psi)$ be a pre-Bayesian game, where $\mathbb{E}=(N, \mathbf{V}, \mathbf{0}, \mathbf{w})$ is the environment. A mediator for $\mathbb{G}$ is a vector $\mathbf{m}=\left(\mathbf{m}_{S}\right)_{S \subseteq N}$, where $\mathbf{m}_{S}: \mathbf{V}_{S} \rightarrow \mathbf{B}_{S}$.

If the set of players that give the mediator the right to play is $S$, and the members of $S$ send the mediator the profile of types $\mathbf{v}_{S}=\left(v_{i}\right)_{i \in S} \in \mathbf{V}_{S}$, the mediator plays $\mathbf{m}_{S}\left(\mathbf{v}_{S}\right) \in \mathbf{B}_{S}$ on behalf of the players in $S$. Every mediator $\mathbf{m}$ for the preBayesian game $\mathbb{G}=(\mathbb{E}, \mathbf{B}, \psi)$ defines a new pre-Bayesian game, $\mathbb{G}^{\mathbf{m}}=\left(\mathbb{E}, \mathbf{B}^{\mathbf{m}}, \psi^{\mathbf{m}}\right)$, which is called the mediated game. This game shares the same environment $\mathbb{E}$ with the original game. The set of actions of $i$ is $B_{i}^{\mathbf{m}}=B_{i} \cup V_{i}$, where without loss of generality we assume that $B_{i} \cap V_{i}=\emptyset$. Choosing $b_{i}^{m}=b_{i} \in B_{i}$ means that $i$ is not using the mediator's services. Choosing $b_{i}^{m}=v_{i} \in V_{i}$ means reporting the value $v_{i}$ to the mediator, as well as the permission to play on behalf of $i$. Let $\mathbf{b}^{\mathbf{m}} \in \mathbf{B}^{\mathbf{m}}$. Denote by $N\left(\mathbf{b}^{\mathbf{m}}\right)$ the set of players that use the mediator; $N\left(\mathbf{b}^{\mathbf{m}}\right)=\left\{i \in N \mid b_{i}^{\mathbf{m}} \in V_{i}\right\}$. The action-to-outcome function in the mediated game, $\psi^{\mathbf{m}}: \mathbf{B}^{\mathbf{m}} \rightarrow \mathbf{0}$, is defined as follows:

$$
\psi^{\mathbf{m}}\left(\mathbf{b}^{\mathbf{m}}\right)=\psi\left(\mathbf{m}_{N\left(\mathbf{b}^{\mathbf{m}}\right)}\left(\mathbf{b}_{N\left(\mathbf{b}^{\mathbf{m}}\right)}^{\mathbf{m}}\right), \mathbf{b}_{-N\left(\mathbf{b}^{\mathbf{m}}\right)}^{\mathbf{m}}\right),
$$

where $-N\left(\mathbf{b}^{\mathbf{m}}\right)=N \backslash N\left(\mathbf{b}^{\mathbf{m}}\right)$. The utility function of $i$ in the mediated game $\mathbb{G}^{\mathbf{m}}$ is denoted by $u_{i}^{\mathbf{m}}$.

Definition 2 (Mediated equilibrium). Let $\mathbb{G}$ be a pre-Bayesian game, and let $\varphi: \mathbf{V} \rightarrow \mathbf{O}$ be an outcome function. We say that $\varphi$ is a mediated equilibrium in $\mathbb{G}$ if there exists a mediator for $\mathbb{G}, \mathbf{m}$, and an ex post equilibrium $\mathbf{g}=\left(g_{1}, \ldots, g_{n}\right)$ in $\mathbb{G}^{\mathbf{m}}$, such that $g_{i}\left(v_{i}\right) \in V_{i}$ for every $i \in N$ and for every $v_{i} \in V_{i}$, and the following holds:

$$
\varphi(\mathbf{v})=\psi\left(\mathbf{m}_{N}(\mathbf{g}(\mathbf{v}))\right), \quad \forall \mathbf{v} \in \mathbf{V} .
$$

The strategy of $i$ at the mediated game in which he gives the right to play to the mediator and reports his true type is called the $T$-strategy. The strategy profile in which every player uses the $T$-strategy is called the $T$-strategy profile. We denote by $\varphi^{\mathbf{m}}: \mathbf{V} \rightarrow \mathbf{O}$ the outcome function generated by the mediator, when every player is using the $T$-strategy. That is,

$$
\varphi^{\mathbf{m}}(\mathbf{v})=\psi\left(\mathbf{m}_{N}(\mathbf{v})\right), \quad \forall \mathbf{v} \in \mathbf{V} .
$$

When the $T$-strategy profile is an ex post equilibrium in $\mathbb{G}^{\mathbf{m}}$ we say that $\mathbf{m}$ implements $\varphi^{\mathbf{m}}$ by truthful mediation. The well known revelation principle ${ }^{11}$ applies to our setting:

Observation 1 (Revelation principle). Let $\mathbb{G}$ be a pre-Bayesian game, and Let $\varphi: \mathbf{V} \rightarrow \mathbf{O}$ be an outcome function. $\varphi$ is a mediated equilibrium if and only if there exists a mediator $\mathbf{m}$ that implements $\varphi$ by truthful mediation. ${ }^{12}$

\section{Position auctions}

In a position auction there is a seller who sells a finite number of positions $j \in K=\{1, \ldots, m\}$. There is a finite number of (potential) bidders $i \in N=\{1, \ldots, n\}$. We assume that there are more bidders than positions, i.e. $n>m$. The positions are sold for a fixed period of time. For each position $j$ there is a commonly-known number $\alpha_{j}>0$, which is interpreted as the expected number of visitors at that position. For every $j \in K \alpha_{j}$ is called the click-through rate of position $j$. We assume that $\alpha_{1}>\alpha_{2}>\cdots>\alpha_{m}>0$. If $i$ holds a position then every visitor to this position gives $i$ a revenue of $v_{i}>0$, where $v_{i}$ is called the valuation of $i$. The set of possible valuations of $i$ is $V_{i}=(0, \infty)$. We assume that the players' payoff functions are quasi-linear. That is, if player $i$ is assigned to position $j$ and pays $p_{j}$ per click, his payoff is $\alpha_{j}\left(v_{i}-p_{j}\right)$. Every player is required to submit a bid, $b_{i} \in B_{i}=[0, \infty)$. We assume that bidding 0 is a symbol for non-participation. Therefore, a player with a bid 0 is not assigned to any position, and pays 0 .

In all position auctions we consider, the player with the highest positive bid receives the first position, the player with the second highest positive bid receives the second position, and so on. ${ }^{13}$ Ties will be considered later. It is useful to define for every position auction two dummy positions, $m+1$ and -1 , which more than one player may be "assigned" to. All players, who participate in the auction but do not get a position in $K$ are assigned to position $m+1$, and all players who choose not to participate are assigned to position -1 . We also define $\alpha_{m+1}=\alpha_{-1}=0$.

An assignment of players to positions is called an allocation. Hence, an allocation is a vector $\mathbf{s}=\left(s_{1}, s_{2}, \ldots, s_{n}\right)$ with $s_{i} \in K \cup\{-1, m+1\}$ such that if $s_{i} \in K, s_{i} \neq s_{l}$ for every $l \neq i ; s_{i}$ is the position of player $i$. The set of all allocations is denoted by $\mathbf{A}$. Given the above, a position auction is defined by its tie breaking rule, which determines the allocation in case of ties, and by its payment scheme. These are discussed below.

\footnotetext{
11 See e.g., Mas-Colell et al. (1995) (page 871).

12 The revelation principle can be generalized as in mechanism design for mediators that enable players to report any message and not just a type.

13 In the proof of our main result we do not use this assumption (see the remarks after Theorem 4).
} 


\subsection{Tie breaking rules}

In practice, the most commonly used tie breaking rule is the first-arrival rule: If a set of players submit the same bid, their priority in receiving the positions is determined by the time their bids were recorded; an earlier bid receives a higher priority. In auction theory this tie breaking rule is typically modeled by assuming that the auctioneer is using a random priority rule. More specifically, let $\Gamma$ be the set of all permutations, $\gamma=\left(\gamma_{1}, \ldots, \gamma_{n}\right)$ of $N=\{1, \ldots, n\}$. Every such $\gamma$ defines a priority rule as follows: Player $i$ has a higher priority than $k$ if and only if $\gamma_{i}<\gamma_{k}$. Every vector of bids $\mathbf{b}$ and a permutation $\gamma$ uniquely determine an allocation. An auctioneer who is using the random priority rule chooses a fixed priority rule $\gamma$ by randomizing uniformly over $\Gamma$. However, the resulting priority rule is not told to the players before they make their bids. When the priority rule $\gamma$ is told to the players before they make their bids, the tie breaking rule is called a fixed priority rule. Dealing with a fixed priority rule simplifies notations and proofs, and in most cases, and in particular in this paper, results that are obtained with this tie breaking rule are identical to the results obtained with the random priority rule. Therefore, unless otherwise specified, we will assume this tie breaking rule.

Without loss of generality we assume that the fixed priority rule is defined by the natural order, $\tilde{\gamma}=(1,2, \ldots, n)$. That is, bidder $i$ has a higher priority than bidder $k$ if and only if $i<k$. Given this fixed priority rule we can make the following definitions, which apply to all position auctions: We denote by $s(\mathbf{b}, i)$ the position player $i$ is assigned to when the bid profile is $\mathbf{b}$. The allocation determined by $\mathbf{b}$ is denoted by

$$
\mathbf{s}(\mathbf{b})=(s(\mathbf{b}, 1), s(\mathbf{b}, 2), \ldots, s(\mathbf{b}, n)) .
$$

For every $j \in K \cup\{-1, m+1\}$ we denote by $\delta(\mathbf{b}, j)$ the set of players assigned to position $j$. Note that for $j \in K, \delta(\mathbf{b}, j)$ contains at most one player. If $\delta(\mathbf{b}, j)$ is a singleton for $j \in K$, we will also refer to it as the player (and not the set with this player) that is assigned to position $j$. To demonstrate these somewhat cumbersome but necessary notations, consider the case $n=7, m=3$, and the following vector of bids $\mathbf{b}=\left(b_{1}, b_{2}, b_{3}, b_{4}, b_{5}, 0,0\right)$ in which $b_{1} \geqslant b_{2} \geqslant b_{3} \geqslant b_{4} \geqslant b_{5}>0$. In this case

$$
s(\mathbf{b})=(1,2,3,4,4,-1,-1), \quad \delta(\mathbf{b}, j)=j, \quad \forall 1 \leqslant j \leqslant 3, \quad \delta(\mathbf{b}, 4)=\{4,5\}, \quad \delta(\mathbf{b},-1)=\{6,7\} .
$$

\subsection{The payment schemes}

Let $\mathbf{B}=B_{1} \times B_{2} \times \cdots \times B_{n}$ be the set of bid profiles. Each position $j \in K \cup\{-1, m+1\}$ is associated with a payment function, $p_{j}: \mathbf{B} \rightarrow \mathbb{R}_{+}$, where $p_{j}(\mathbf{b})$ is the payment per click for position $j$ when the bid profile is $\mathbf{b}$. Naturally we assume that $p_{-1}$ is identically zero. However, we also assume that $p_{m+1}$ is identically zero. Hence, a participant who is not assigned a real position pays nothing. The vector of payment functions $\mathbf{p}=\left(p_{j}\right)_{j \in K}$ is called the position payment scheme. We will make the following two assumptions on the position payment scheme:

- Position payment functions are anonymous, i.e. the players' payments to the auctioneer are not influenced by their identities. More precisely: Let $\mathbf{b} \in \mathbf{B}$ be a bid profile. We denote by $b_{(j)}$ the $j$ th highest bid in $\mathbf{b}$. For example if $\mathbf{b}=(3,7,3,0,2)$ then $b_{(1)}=7, b_{(2)}=3, b_{(3)}=3, b_{(4)}=2, b_{(5)}=0$. We let $\mathbf{b}^{*}=\left(b_{(1)}, \ldots, b_{(n)}\right)$. Anonymity is modeled by the requirement that for every two bid profiles $\mathbf{b}, \mathbf{d} \in \mathbf{B}, \mathbf{p}(\mathbf{b})=\mathbf{p}(\mathbf{d})$ whenever $\mathbf{b}^{*}=\mathbf{d}^{*}$.

- A player never pays more than his bid. That is, $p_{j}(\mathbf{b}) \leqslant b_{(j)}$ for every $\mathbf{b} \in \mathbf{B}$ and for every $j \in K$.

It is convenient in certain cases to describe the payment functions indexed by the players. Let $\mathbb{G}$ be a position auction with a position payment scheme $\mathbf{p}$.

For every player $i$ we denote

$$
q_{i}(\mathbf{b})=p_{s(\mathbf{b}, i)}(\mathbf{b}),
$$

and

$$
\mathbf{q}(\mathbf{b})=\left(q_{1}(\mathbf{b}), q_{2}(\mathbf{b}), \ldots q_{n}(\mathbf{b})\right) .
$$

Note that the correspondence $\mathbf{p} \rightarrow \mathbf{q}$ is one-to-one. We call $\mathbf{q}$ the player payment scheme. All our assumptions about the position payment schemes can be transformed to analogous assumptions about the player payment schemes. We will describe position auctions by their position payment schemes, $\mathbf{p}$, but we will freely use the associated player payment scheme, $\mathbf{q}$, in the definitions and proofs.

\subsection{Central position auctions}

We next describe the payment schemes of three central position auctions.

Self-price position auctions: Each player who is assigned to a position with a positive click-through rate pays his own bid. That is, for every $j \in K$ and every $\mathbf{b} \in \mathbf{B}$

$$
p_{j}(\mathbf{b})=b_{(j)} .
$$


Next-price position auctions: In this auction (run with a slight variation by Google), every player who is assigned to a position with a positive click-through rate pays the bid of the player assigned to the position right after him if there is such a player, and zero otherwise. That is, for every $j \in K$ and for every $\mathbf{b} \in B$

$$
p_{j}(\mathbf{b})=b_{(j+1)} \text {. }
$$

VCG position auctions: In a Vickrey-Clarke-Groves (VCG) position auction the payment function for position $j \in K$ is defined as follows ${ }^{14}$ : For every $\mathbf{b} \in \mathbf{B}$

$$
p_{j}^{v c g}(\mathbf{b})=\frac{\sum_{k=j+1}^{m+1} b_{(k)}\left(\alpha_{k-1}-\alpha_{k}\right)}{\alpha_{j}} .
$$

\subsection{Position auctions as pre-Bayesian games}

We denote by $\mathbb{G}=\mathbb{G}(\alpha, \mathbf{p})$ the position auction with the click-through rate vector $\alpha$ and the position payment scheme p. We denote by $\mathbf{q}$ the associated player payment scheme. $\mathbb{G}$ is a pre-Bayesian game as defined in Section 2 :

$N=\{1, \ldots, n\}$ is the set of players.

$V_{i}=(0, \infty)$ is the set of types of $i$, and $\mathbf{V}=V_{1} \times V_{2} \times \cdots \times V_{n}$ is the set of states.

$B_{i}=[0, \infty)$ is the set of actions of $i$, and $\mathbf{B}=B_{1} \times B_{2} \times \cdots \times B_{n}$ is the set of action profiles.

$\mathbf{O}=\mathbf{A} \times \mathbb{R}_{+}^{n}$ is the set of outcomes, where $\mathbf{A}$ is the set of allocations; a typical outcome is $\left(s_{1}, \ldots, s_{n}, c_{1}, \ldots, c_{n}\right)$, where $s_{i}$ is the slot of $i$, and $c_{i}$ is the cost per click paid by $i$. Position auctions are not as general as the pre-Bayesian games defined in Section 2: The payoff function of $i$ depends only on $v_{i}$ and not on the vector of types $\mathbf{v}=\left(v_{1}, \ldots, v_{n}\right)$. Hence, $w_{i}: V_{i} \times \mathbf{O} \rightarrow \mathbb{R}$ is defined as follows:

$$
w_{i}\left(v_{i},(\mathbf{s}, \mathbf{c})\right)=w_{i}\left(v_{i},\left(s_{1}, \ldots, s_{n}, c_{1}, \ldots, c_{n}\right)\right)=\alpha_{s_{i}}\left(v_{i}-c_{i}\right) .
$$

The function that maps profiles of actions to outcomes, $\psi: \mathbf{B} \rightarrow \mathbf{O}$ is

$$
\psi(\mathbf{b})=(s(\mathbf{b}), \mathbf{q}(\mathbf{b})) .
$$

The utility function for player $i, u_{i}: V_{i} \times \mathbf{B} \rightarrow \mathbb{R}$ is therefore defined as follows:

$$
u_{i}\left(v_{i}, \mathbf{b}\right)=\alpha_{s(\mathbf{b}, i)}\left(v_{i}-q_{i}(\mathbf{b})\right) .
$$

\subsection{Mediators for position auctions}

We next recall the definitions from Section 2, and apply them to the context of position auctions. Let $\mathbb{G}$ be a position auction. A mediator for $\mathbb{G}$ is a vector of functions $\mathbf{m}=\left(\mathbf{m}_{S}\right)_{S \subseteq N}$, where $\mathbf{m}_{S}: \mathbf{V}_{S} \rightarrow \mathbf{B}_{S}$. The mediator $\mathbf{m}$ generates the preBayesian mediated game $\mathbb{G}^{\mathbf{m}}$. In this game every player $i$ receives his type $v_{i}$ and can either send a type, $\hat{v}_{i}$ (not necessarily the true type) to the mediator, or submit a bid directly to the auction. If $S$ is the set of players that send a type to the mediator, the mediator bids on their behalf $\mathbf{m}_{S}\left(\hat{\mathbf{v}}_{S}\right)$. Hence, the action set of player $i$ in the mediated game is $B_{i} \cup V_{i}$, where in this case $V_{i}$ is a copy $(0, \infty)$ which is disjoint from $B_{i} \cdot{ }^{15}$ Recall that the $T$-strategy for a player in the mediated game is the strategy in which the player uses the mediator's services and reports to her his true type. The $T$-strategy profile is the profile of strategies in which every player is using the $T$-strategy.

Definition 3. The $T$-strategy profile is an ex post equilibrium in the mediated game if for every player $i$ and type $v_{i}$, and for every vector of types of the other players, $\mathbf{v}_{-i}$, the following two conditions hold:

E1: $i$ is not better off when he gives the mediator the right to play and report a false type. That is, for every $\hat{v}_{i} \in V_{i}$

$$
u_{i}\left(v_{i}, \mathbf{m}_{N}\left(v_{i}, \mathbf{v}_{-i}\right)\right) \geqslant u_{i}\left(v_{i}, \mathbf{m}_{N}\left(\hat{v}_{i}, \mathbf{v}_{-i}\right)\right) \text {. }
$$

E2: $i$ is not better off when he bids directly. That is, for every $b_{i} \in B_{i}$,

$$
u_{i}\left(v_{i}, \mathbf{m}_{N}\left(v_{i}, \mathbf{v}_{-i}\right)\right) \geqslant u_{i}\left(v_{i}, b_{i}, \mathbf{m}_{N \backslash\{i\}}\left(\mathbf{v}_{-i}\right)\right) .
$$

Whenever the $T$-strategy profile is an ex post equilibrium in $\mathbb{G}^{\mathbf{m}}$, the mediator $\mathbf{m}$ implements an outcome function in $\mathbb{G}$. This outcome function is denoted by $\varphi^{\mathbf{m}}: \mathbf{V} \rightarrow \mathbf{0}$, and it is defined as follows:

$$
\varphi^{\mathbf{m}}(\mathbf{v})=\psi\left(\mathbf{m}_{N}(\mathbf{v})\right)=\left(\mathbf{s}\left(\mathbf{m}_{N}(\mathbf{v})\right), \mathbf{q}\left(\mathbf{m}_{N}(\mathbf{v})\right)\right) .
$$

\footnotetext{
14 We use the standard payment function of the VCG mechanism. A general VCG mechanism may be obtained from the standard one by adding an additional payment function to each player, which depends only on the declarations of the other players. Some authors (see e.g., Holzman et al., 2004) call the standard VCG mechanism, the VC mechanism. According to this terminology we actually deal with VC position auctions. However, we decided to use the more common terminology.

15 A player either chooses a bid, when participating directly in the auction, or a type, when using the mediator's services.
} 


\subsection{Implementing the VCG outcome function by truthful mediation}

As is discussed in the introduction, in this paper we deal with implementation of the outcome of the VCG position auction by truthful mediation. In this section we demonstrate this type of implementation by an example. This example also shows the need for a definition of individually rational mediators.

Given a position auction $\mathbb{G}$ our goal is to construct a mediator that would implement the outcome function of the VCG position auction. This outcome function, $\varphi^{v c g}: \mathbf{V} \rightarrow \mathbf{0}$ is defined as follows:

$$
\varphi^{v c g}(\mathbf{v})=\left(\mathbf{s}(\mathbf{v}), \mathbf{q}^{v c g}(\mathbf{v})\right)
$$

Definition 4. Let $\mathbb{G}$ be a position auction. Let $\mathbf{m}$ be a mediator for $\mathbb{G}$. We say the $\mathbf{m}$ implements the VCG outcome function in $\mathbb{G}$, or that it implements $\varphi^{v c g}$ in $\mathbb{G}$, if the $T$-strategy profile is an ex post equilibrium in $\mathbb{G}^{\mathbf{m}}$, and $\varphi^{\mathbf{m}}=\varphi^{v c g}$.

We demonstrate the implementation of the VCG outcome function by a simple example:

Example 1. Consider a self-price position auction $\mathbb{G}=\mathbb{G}(\alpha, \mathbf{p})$ with two players and one position, and with $\alpha_{1}=1$. That is, $\mathbb{G}$ is a standard two-person first-price auction. The corresponding VCG position auction is a standard second-price auction. We define a family of mediators $\mathbf{m}^{c}, c \geqslant 1$, each of them implements the VCG position auction. Assume both players use the mediator's services and send it the types $\hat{\mathbf{v}}=\left(\hat{v}_{1}, \hat{v}_{2}\right)$, then the $c$-mediator acts as follows: If $\hat{v}_{1} \geqslant \hat{v}_{2}$ the mediator makes the following bids on behalf of the players: $b_{1}=\hat{v}_{2}$, and $b_{2}=\frac{\hat{v}_{2}}{2}$. If $\hat{v}_{2}>\hat{v}_{1}$, the mediator makes the bids $b_{1}=\frac{\hat{v}_{1}}{2}, b_{2}=\hat{v}_{1}$. If only one player uses the mediator's services, say player $i$, the mediator bids $b_{i}=c \hat{v}_{i}$ on behalf of $i$. We claim that for every $c \geqslant 1$, the $T$-strategy profile is an ex post equilibrium in the mediated game generated by $\mathbf{m}^{c}$. Indeed, assume player 2 uses the $T$-strategy and reports his type $v_{2}$ to the mediator, and consider player 1 . If $v_{1} \geqslant v_{2}$ then by using the $T$-strategy player 1 receives the position and pays $v_{2}$. Hence, 1 's utility is $v_{1}-v_{2}$. If player 1 deviates by using the mediator's services and reporting $\hat{v}_{1} \geqslant v_{2}$ his utility is still $v_{1}-v_{2}$. If he reports $\hat{v}_{1}<v_{2}$ his utility will be 0 . If player 1 does not use the mediator, he should bid at least $c v_{2}$ in order to get the position, and therefore his utility cannot exceed $v_{1}-v_{2}$. If $v_{1}<v_{2}$, then the $T$-strategy yields 0 , and any other strategy yields a non-positive utility. Hence, the $T$-strategy profile is an ex post equilibrium for every $c$-mediator, $c \geqslant 1 .^{16}$

While each of the mediators $\mathbf{m}^{c}, c \geqslant 1$, in Example 1 implements the VCG outcome function, the mediator with $c=1$ has a distinct characteristic: a player who uses the $T$-strategy cannot get a negative utility. In contrast, for every $c>1$, if say player 2 does not use the mediator's services, participates directly and bids less than $c v_{1}$, then the $T$-strategy yields a negative utility of $(1-c) v_{1}$ to player 1 . This motivates our definition of individually rational mediators:

Definition 5 (Individually rational mediator). Let $\mathbb{G}$ be a position auction. A mediator $\mathbf{m}$ for $\mathbb{G}$ is individually rational, if for every player, using the $T$-strategy guarantees a non-negative level of utility. That is, for every $S \subseteq N$ and every player $i \in S$

$$
u_{i}\left(v_{i}, \mathbf{m}_{S}\left(\mathbf{v}_{S}\right), \mathbf{b}_{-S}\right) \geqslant 0, \quad \forall \mathbf{b}_{-S} \in \mathbf{B}_{-S} \text { and } \forall \mathbf{v}_{S} \in \mathbf{V}_{S}
$$

Hence, in Example 1 the mediator $\mathbf{m}^{c}$, with $c=1$, is an individually rational mediator that implements the VCG outcome function. ${ }^{17}$

\section{Mediators in next-price position auctions}

In this section we show that there exists an individually rational mediator, which implements the VCG outcome function in next-price position auctions. Although in the following section we prove a more general result, we present this result first, given the importance of next-price position auctions in the literature and in practice.

Theorem 2. Let $\mathbb{G}$ be a next-price position auction. There exists an individually rational mediator that implements $\varphi^{v c g}$ in $\mathbb{G}$.

In order to prove Theorem 2 and other theorems we need the following lemma:

Lemma 3. Let $\mathbf{p}^{v c g}$ be the VCG payment scheme.

1. $p_{j}^{v c g}(\mathbf{b}) \leqslant b_{(j+1)}$ for every $j \in K$.

\footnotetext{
${ }^{16}$ However, note that the $T$-strategy is not a dominant strategy; e.g., for $c>1$, if $v_{1}>v_{2}$ and player 2 bids directly $v_{2}$ (without using the mediator services), then bidding directly $\frac{v_{1}+v_{2}}{2}$ is better for player 1 than using the $T$-strategy: in the former case player 1 's utility is $\frac{v_{1}-v_{2}}{2}$ and in the latter case her utility is non-positive. A similar argument shows that the $T$-strategy is not dominant for $c=1$ as well.

17 It is interesting to note that this simple example cannot be extended to general (more than one slot) self-price position auctions, as will be discussed in Section 6.
} 
2. $p_{j}^{v c g}(\mathbf{b}) \geqslant p_{j+1}^{v c g}(\mathbf{b})$ for every $j=1, \ldots, m-1$ and for every $\mathbf{b} \in B$, where for every $j$, equality holds if and only if $b_{(j+1)}=b_{(j+2)}=$ $\cdots=b_{(m+1)}$.

The proof of Lemma 3 is given in Section 8. In the proof of Theorem 2 we will show that the following mediator implements $\varphi^{v c g}$ in a next-price position auction ${ }^{18}$ :

Mediator 1 (A mediator for next-price position auctions).

(a) For every $\mathbf{v} \in V$ let $\mathbf{m}_{N}(\mathbf{v})=\mathbf{b}(\mathbf{v})$, where $\mathbf{b}(\mathbf{v})$ is defined as follows:

- $\mathbf{b}_{i}(\mathbf{v})=p_{s(\mathbf{v}, i)-1}^{v c g}(\mathbf{v})$ for every player $i$ such that $2 \leqslant s(\mathbf{v}, i) \leqslant m .^{19}$

- $\mathbf{b}_{i(\mathbf{v})}(\mathbf{v})=p_{m}^{v c g}(\mathbf{v})$, where $i(\mathbf{v})$ is the player with the highest index in $\delta(\mathbf{v}, m+1)$.

- $\mathbf{b}_{i}(\mathbf{v})=\frac{\mathbf{b}_{i(\mathbf{v})}(\mathbf{v})}{1+\rho}$ for every $i \in \delta(\mathbf{v}, m+1) \backslash\{i(\mathbf{v})\}$ for some arbitrary but fixed $\rho>0$.

- $\mathbf{b}_{\delta(\mathbf{v}, 1)}(\mathbf{v})=\epsilon+p_{1}^{v c g}(\mathbf{v})$ for some arbitrary but fixed $\epsilon>0 .{ }^{20}$

(b) For every strict subset $S \subset N \mathbf{m}_{S}(\mathbf{v})=\mathbf{v}_{S}$ for every $\mathbf{v}_{S} \in V_{S}$.

We first give a short intuitive explanation of why Mediator 1 implements the VCG outcome function when every player uses the $T$-strategy. Assume $v_{1}>\cdots>v_{m}>\cdots>v_{n}$. In the VCG position auction, player $i$ receives position $i$ for every $1 \leqslant i \leqslant m$, and such a player $i$ pays $p_{i}^{v c g}(\mathbf{v})$. All players $i, i \geqslant m+1$, are assigned to the dummy position, $m+1$, and pay nothing. The vector of bids submitted to the next-price position auction is $\mathbf{b}(\mathbf{v})=\left(b_{1}(\mathbf{v}), \ldots, b_{n}(\mathbf{v})\right)$, where

$$
\mathbf{b}(\mathbf{v})=\left(\epsilon+p_{1}^{v c g}(\mathbf{v}), p_{1}^{v c g}(\mathbf{v}), p_{2}^{v c g}(\mathbf{v}), \ldots, p_{m-1}^{v c g}(\mathbf{v}), \frac{p_{m}^{v c g}(\mathbf{v})}{1+\rho}, \frac{p_{m}^{v c g}(\mathbf{v})}{1+\rho}, \ldots, \frac{p_{m}^{v c g}(\mathbf{v})}{1+\rho}, p_{m}^{v c g}(\mathbf{v})\right) .
$$

By the second part of Lemma $3, p_{1}^{v c g}(\mathbf{v})>p_{2}^{v c g}(\mathbf{v})>p_{m}^{v c g}(\mathbf{v})$, and therefore $b_{1}(\mathbf{v})>b_{2}(\mathbf{v})>\cdots>b_{m}(\mathbf{v})>b_{i}(\mathbf{v})$ for every $m+1 \leqslant i \leqslant n$. Hence the vector $\mathbf{b}(\mathbf{v})$ submitted to the next-price position auction generates the same allocation as the vector $\mathbf{v}$ submitted to the VCG position auction. Moreover, when $\mathbf{b}(\mathbf{v})$ is submitted to the next-price position auction, every player $i, 1 \leqslant i \leqslant m$, pays $p_{i}^{v c g}(\mathbf{v})$. Hence, the vector $\mathbf{b}(\mathbf{v})$ submitted to the next-price position auction generates the same outcome as the vector $\mathbf{v}$ submitted to the VCG position auction, hence $\varphi^{\mathbf{m}}(\mathbf{v})=\varphi^{v c g}(\mathbf{v})$, where $\mathbf{m}$ is Mediator 1 . Additional complications arise when there are ties in the vector $\mathbf{v}$. These complications, as well as the proof that the $T$-strategy profile is an ex post equilibrium in the mediated game, and that Mediator 1 is individually rational are treated in the full proof below.

Proof of Theorem 2. Let $\mathbf{m}$ be Mediator 1 defined above. We first show that $\varphi^{\mathbf{m}}(\mathbf{v})=\varphi^{v c g}(\mathbf{v})$ for every $\mathbf{v} \in V$. Let $\mathbf{v} \in V$ be an arbitrary valuation vector. We need to show that $s(\mathbf{b}(\mathbf{v}))=s(\mathbf{v})$ and that $\mathbf{q}(\mathbf{b}(\mathbf{v}))=\mathbf{q}^{v c g}(\mathbf{v})$, where $\mathbf{q}$ is the player payment scheme in the next-price position auction. We begin by showing that $s(\mathbf{b}(\mathbf{v}))=s(\mathbf{v})$. Let $i \neq l$ be a pair of players. It suffices to show that whenever $1 \leqslant s(\mathbf{v}, i)<s(\mathbf{v}, l) \leqslant m+1, s(\mathbf{b}(\mathbf{v}), i)<s(\mathbf{b}(\mathbf{v}), l)$.

Case 1: $s(\mathbf{v}, i)=1$. In this case $i=\delta(\mathbf{v}, 1)$, and therefore $b_{i}(\mathbf{v})=\epsilon+p_{1}^{v c g}(\mathbf{v})$. On the other hand, $b_{l}(\mathbf{v}) \leqslant \max _{k=1}^{n} p_{k}^{v c g}(\mathbf{v})$. Therefore, by the second part of Lemma $3, b_{i}(\mathbf{v})>b_{l}(\mathbf{v})$. Hence, $s(\mathbf{b}(\mathbf{v}), i)<s(\mathbf{b}(\mathbf{v}), l)$.

Case 2: $s(\mathbf{v}, i)>1, s(\mathbf{v}, l)=m+1$, and $l \neq i(\mathbf{v})$. Since $l$ gets the dummy slot, $m+1$, and $l \neq i(\mathbf{v}), b_{l}(v)=\frac{p_{m}^{v c g}(v)}{\rho}<p_{m}^{v c g}(v)$. On the other hand, $i$ gets a real slot, and therefore, by the second part of Lemma $3, b_{i}(v) \geqslant p_{m}^{v c g}(v)$. Hence, $\mathbf{b}_{l}(\mathbf{v})<\mathbf{b}_{i}(\mathbf{v})$, implying that $s(\mathbf{b}(\mathbf{v}), i)<s(\mathbf{b}(\mathbf{v}), l)$.

Case 3: $s(\mathbf{v}, i)>1$, and $[l \leqslant m$ or $l=i(\mathbf{v})]$. We distinguish between two cases:

1. $v_{i}=v_{l}$. Since $s(\mathbf{v}, i)<s(\mathbf{v}, l)$, the fixed priority rule implies that $i<l$. By the second part of Lemma $3, p_{s(\mathbf{v}, i)-1}^{v c g}(\mathbf{v}) \geqslant$ $p_{s(\mathbf{v}, l)-1}^{v c c}(\mathbf{v})$. Therefore, $\mathbf{b}_{i}(\mathbf{v}) \geqslant \mathbf{b}_{l}(\mathbf{v})$, which implies that $s(\mathbf{b}(\mathbf{v}), i)<s(\mathbf{b}(\mathbf{v}), l)$.

2. $v_{i}>v_{l}$. By the second part of Lemma $3, p_{s(\mathbf{v}, i)-1}^{v c g}(\mathbf{v})>p_{s(\mathbf{v}, i)}^{v c g}(\mathbf{v})$. Since $s(\mathbf{v}, i) \leqslant s(\mathbf{v}, l)-1$, by the second part of Lemma 3 , $p_{s(\mathbf{v}, i)}^{v c g}(\mathbf{v}) \geqslant p_{s(\mathbf{v}, l)-1}^{v c g}(\mathbf{v})$. Therefore, $p_{s(\mathbf{v}, i)-1}^{v c g}(\mathbf{v})>p_{s(\mathbf{v}, l)-1}^{v c g}(\mathbf{v})$, implying that $\mathbf{b}_{i}(\mathbf{v})>\mathbf{b}_{l}(\mathbf{v})$. Therefore $s(\mathbf{b}(\mathbf{v}), i)<s(\mathbf{b}(\mathbf{v}), l)$.

This completes the proof that $s(\mathbf{b}(\mathbf{v}))=s(\mathbf{v})$ for all $\mathbf{v} \in V$.

Observe that for every player $i$ for which $s(\mathbf{b}(\mathbf{v}), i) \in K, p_{s(\mathbf{b}(\mathbf{v}), i)}(\mathbf{b}(\mathbf{v}))=p_{s(\mathbf{v}, i)}^{v c g}(\mathbf{v})$, where $\mathbf{p}=\left(p_{j}\right)_{j=1}^{m}$ is the position payment scheme in the next-price position auction. Therefore, $q_{i}(\mathbf{b}(\mathbf{v}))=q_{i}^{v c g}(\mathbf{v})$ for every $i \in N$. This shows that $\mathbf{q}(\mathbf{b}(\mathbf{v}))=$ $\mathbf{q}^{v c g}(\mathbf{v})$ for all $\mathbf{v} \in V$. Hence, $\varphi^{\mathbf{m}}=\varphi^{v c g}$.

\footnotetext{
18 For the case in which all players choose the mediator, the mediator is similar to the algorithm given in Edelman et al. (2007).

19 Recall that $s(\mathbf{b}, i)$ denotes the position of player $i$ under the bid profile $\mathbf{b}$.

20 Recall that whenever $j \in K=\{1, \ldots, m\}$ (the set of non-dummy positions), $\delta(\mathbf{b}, j$ ) denotes the player assigned to position $j$.
} 
We proceed to prove that the $T$-strategy profile is an ex post equilibrium. Let $\mathbf{v} \in V$ and suppose every player but $i$ uses the $T$-strategy. Reporting to the mediator a false value is not beneficial for $i$ by the truthfulness of VCG. Assume that player $i$ participates directly in the auction. Hence, $\mathbf{v}_{-i}$ is the vector of bids submitted by the mediator. Let $b_{i}$ be player $i$ 's bid. Let $k=s(\mathbf{v}, i)$. Since $\varphi^{\mathbf{m}}=\varphi^{v c g}, s(\mathbf{b}(\mathbf{v}), i)=k$. Let $j$ be the position of player $i$ after the deviation, that is $j=s\left(\left(\mathbf{v}_{-i}, b_{i}\right), i\right)$. If $j \notin K$, the utility of player $i$ is zero, and therefore deviating is not profitable for $i$. Suppose $j \in K$. Let $\tilde{\mathbf{b}}=\left(\mathbf{v}_{-i}, b_{i}\right)$. Observe that

$$
\alpha_{k}\left(v_{i}-p_{k}(\mathbf{b}(\mathbf{v}))\right)=\alpha_{k}\left(v_{i}-p_{k}^{v c g}(\mathbf{v})\right) \geqslant \alpha_{j}\left(v_{i}-p_{j}^{v c g}(\tilde{\mathbf{b}})\right) \geqslant \alpha_{j}\left(v_{i}-\tilde{b}_{(j+1)}\right),
$$

where the first equality follows since $\varphi^{\mathbf{m}}=\varphi^{v c g}$, the first inequality follows since VCG is truthful, and the second inequality follows from the first part of Lemma 3. Since $p_{j}$ is position $j$ 's payment function in the next-price position auction, $\alpha_{j}\left(v_{i}-\right.$ $\left.\tilde{b}_{(j+1)}\right)=\alpha_{j}\left(v_{i}-p_{j}(\tilde{\mathbf{b}})\right)$. Therefore,

$$
\alpha_{k}\left(v_{i}-p_{k}(\mathbf{b}(\mathbf{v}))\right) \geqslant \alpha_{j}\left(v_{i}-p_{j}(\tilde{\mathbf{b}})\right) .
$$

Hence, player $i$ does not gain from participating directly in the auction.

Finally, we show that $\mathbf{m}$ is individually rational. Let $i$ be a player that uses the $T$-strategy whose valuation is $v_{i}$. If all other players choose the mediator and report $\mathbf{v}_{-i}$, the generated outcome in the next-price position auction is precisely the outcome generated in the VCG position auction by the vector of valuations $\mathbf{v}$. Therefore, by the first part of Lemma 3 player $i$ does not pay more than $v_{i}$. Consider the situation in which a subset of players, $S, i \notin S$, participates directly in the auction. Since $i$ uses the $T$-strategy, the mediator submits $i$ 's value to the next-price position auction, and therefore, according to the rules of the next-price position auction, $i$ will not pay more than her value.

\section{Implementing the VCG outcome function in general position auctions}

\subsection{Main result}

In the previous section we discussed the implementation of the VCG outcome function in the next-price position auction. In this section we provide a much larger class of position auctions in which it is possible to implement $\varphi^{v c g}$ by an individually rational mediators. We are about to give sufficient conditions for implementing $\varphi^{v c g}$ by an individually rational mediator in position auctions. We need the following definitions:

Definition 6 (GLP position auctions). A position auction $\mathbb{G}$ is a generalized lower price (GLP) position auction, if the payment of each player who is assigned to a position in $K$, is a function of the bids of players assigned to "lower" positions (that is, positions with higher indices) than his own. More specifically, for every $j \in K$ and for every two bid profiles $\mathbf{b}^{1}, \mathbf{b}^{2} \in \mathbf{B}$ such that $b_{(l)}^{1}=b_{(l)}^{2}$ for every $l>j$,

$$
p_{j}\left(\mathbf{b}^{1}\right)=p_{j}\left(\mathbf{b}^{2}\right) .
$$

Definition 7 (VCG cover). A position auction $\mathbb{G}$ is a VCG cover if for every $\mathbf{v} \in \mathbf{V}$ there exists $\mathbf{b} \in \mathbf{B}$ such that $\psi^{\mathbb{G}}(\mathbf{b})=\varphi^{v c g}(\mathbf{v})$, where $\psi^{\mathbb{G}}(\mathbf{b})=(s(\mathbf{b}), \mathbf{q}(\mathbf{b}))$.

Definition 8 (Monotone position auctions). A position auction $\mathbb{G}$ is monotone if $p_{j}(\mathbf{b}) \geqslant p_{j}\left(\mathbf{b}^{\prime}\right)$ for every $j \in K$ and for every $\mathbf{b} \geqslant \mathbf{b}^{\prime}$, where $\mathbf{b} \geqslant \mathbf{b}^{\prime}$ if and only if $b_{i} \geqslant b_{i}^{\prime}$ for every $i \in N$.

We are now able to show:

Theorem 4. Let $\mathbb{G}$ be a position auction.

(i) If the following three conditions hold, there exists an individually rational mediator that implements $\varphi^{v c g}$ in $\mathbb{G}$ :

1. $\mathbb{G}$ is a GLP position auction.

2. $\mathbb{G}$ is a VCG cover.

3. $\mathbb{G}$ is monotone.

(ii) The set of conditions 1-3 is minimal. That is, if any one of the conditions 1-3 is dropped, there exists a position auction which satisfies the two other conditions, but $\varphi^{v c g}$ cannot be implemented by an individually rational mediator.

The following remarks relate to part (i) of Theorem 4 . 


\section{Remarks.}

1. Theorem 4 applies in particular to next-price position auctions discussed in Section 4 . Hence, it implies Theorem 2. However, the mediator constructed for this general case, Mediator 2, is different from Mediator 1 used in the proof of Theorem 2. Moreover, Theorem 4 applies to many other interesting position auctions as will be shown later.

2. The monotonicity condition is only used when proving that Mediator 2 is individually rational.

3. In this paper we assume that the allocation rule ranks players in positions in decreasing order of their bids. We actually prove a stronger result than stated in Theorem 4. All we require from the allocation rule of the position auction $\mathbb{G}$ in Theorem 4 is that if a single player changes her bid, the relative order of the other players is "almost" unaffected; more precisely, if a single player $i$ changes her bid from $b_{i}$ to $b_{i}^{\prime}$, for every other pair of players $k, l$ the following holds: $s_{k}\left(b_{i}, b_{-i}\right)>s_{l}\left(b_{i}, b_{-i}\right)$ implies $s_{k}\left(b_{i}^{\prime}, b_{-i}\right) \geqslant s_{l}\left(b_{i}^{\prime}, b_{-i}\right)$.

The proof of part (i) of Theorem 4 is given in Section 8, and the proof of part (ii) is given in the next subsection. In what follows, we describe the mediator constructed for the proof of part (i) and provide some intuition for why this mediator implements the VCG outcome.

For simplicity, in this discussion we assume there are no ties. Since $\mathbb{G}$ is a VCG cover, for every valuation profile $\mathbf{v} \in \mathbf{V}$ there exist a bid profile $\mathbf{b}(\mathbf{v})$ that yields in $\mathbb{G}$ the same outcome generated in the VCG position auction when the bid profile is $\mathbf{v}$. Consider the following mediator $\mathbf{m}$ : if all players use the mediator's services and submit the vector of valuations $\mathbf{v}$, the mediator submits on their behalf $\mathbf{b}(\mathbf{v})$; if all players but $i$ use the mediator's services and submit $\mathbf{v}_{-i}$, the mediator behave as if $i$ has the largest value and submits the appropriate bids on behalf of the players in $N \backslash\{i\}$. More precisely, the mediator submits $\mathbf{b}_{-i}\left(\mathbf{v}^{i}\right)$, where $\mathbf{v}^{i}$ is the bid profile obtained from $\mathbf{v}$ by replacing $v_{i}$ with a value larger than all other players' values; if the mediator receives the profile $\mathbf{v}_{S}$ for some subset of the players containing less than $n-1$ valuations, the mediator redirects the valuations to the auction. To see why the mediator implements the VCG outcome, assume only player $i$ does not use the $T$-strategy. Obviously reporting falsely to the mediator is not beneficial for $i$ since VCG is truthful. Suppose $i$ participates directly in the auction, bids $b_{i}$, and is assigned to position $j$. There exists some value $\tilde{v}_{i}$ such that reporting this value to the mediator yields player $i$ position $j$. Therefore, since $\mathbf{m}$ pretends $i$ has the highest value when $i$ does not use its services and $\mathbb{G}$ is a GLP position auction, $i$ will pay the same amount regardless of whether she bids $b_{i}$ directly or submits $\tilde{v}_{i}$ to the mediator. However, reporting $\tilde{v}_{i}$ to the mediator is not beneficial for $i$, and participating directly is also not beneficial for $i$. The key in proving that $\mathbf{m}$ is individually rational is to show that a single deviator can cause each of the other players to move only a single position up or down, which will not harm her payoff "too much." This part is more involved, and we leave it to the full proof.

\subsection{Part (ii) of Theorem 4}

In his subsection we prove part (ii) of Theorem 4 . Obviously, in order to implement $\varphi^{v c g}$ by truthful mediation $\mathbb{G}$ must be a VCG cover. We prove the necessity of conditions 1 and 3 through Examples 2 and 3, respectively.

Example 2 (GLP is necessary). Let $\mathbb{G}=\mathbb{G}(\alpha, \mathbf{p})$ be the following position auction: $N=\{1,2,3\}, K=\{1,2\}$, and $\alpha=(2,1)$ Let $p_{1}(\mathbf{b})=\frac{b_{(1)}}{4}$ and $p_{2}(\mathbf{b})=b_{(2)}$. Observe that $\mathbb{G}$ is monotone. We next show that $\mathbb{G}$ is a VCG cover. Let $\mathbf{v} \in \mathbf{V}$ be an arbitrary valuation profile. We need to show that there exists a bid profile $\mathbf{b}(\mathbf{v})$ such that $\psi^{\mathbb{G}}(\mathbf{b}(\mathbf{v}))=\varphi^{v c g}(\mathbf{v})$. Note that $p_{1}^{v c g}(\mathbf{v})=\frac{v_{(2)}+v_{(3)}}{2}$ and $p_{2}^{v c g}(\mathbf{v})=v_{(3)}$. Let $\mathbf{b}(\mathbf{v})$ be the following bid profile: $b_{\delta(\mathbf{v}, 3)}(\mathbf{v})=\frac{v_{(3)}}{2}, b_{\delta(\mathbf{v}, 2)}(\mathbf{v})=v_{(3)}$ and $b_{\delta(\mathbf{v}, 1)}(\mathbf{v})=$ $2 v_{(2)}+2 v_{(3)}$. By construction, $s(\mathbf{b}(\mathbf{v}), i)=s(\mathbf{v}, i)$ for $i=1,2,3$. In addition, observe that $p_{j}(\mathbf{b}(\mathbf{v}))=p_{j}^{v c g}(\mathbf{v})$ for $j=1,2$. Therefore, $\psi^{\mathbb{G}}(\mathbf{b}(\mathbf{v}))=\varphi^{v c g}(\mathbf{v})$, implying that $\mathbb{G}$ is a VCG cover. Note however that $\mathbb{G}$ is not a GLP position auction. Assume for contradiction that there exists an individually rational mediator $\mathbf{m}$, which implements the VCG outcome function in $\mathbb{G}$. Consider the vector of valuations $\mathbf{v}=(12,10,8)$. If all players use the $T$-strategy, player 2 (with valuation 10$)$ gets position 2 , pays 8 , and therefore her utility is $1(10-8)=2$. Player 2 can always bid more than the other players, and by that cause some other player to be positioned second; therefore, since $\mathbf{m}$ is individually rational it cannot submit more than 12 on behalf of either of the players 1 and 3. Hence, if both players, 1 and 3, use the $T$-strategy then by deviating and bidding 13 , player 2 gets the first position and here utility is $2\left(10-\frac{13}{4}\right)>2$, yielding a contradiction.

Example 3 (Monotonicity is necessary). Let $\mathbb{G}=\mathbb{G}(\alpha, \mathbf{p})$ be the following position auction: $N=\{1,2,3,4\}, K=\{1,2,3\}$ $\alpha=(100,10,1), p_{1}(\mathbf{b})=b_{(2)}-b_{(3)}, p_{2}(\mathbf{b})=\frac{b_{(3)}+b_{(4)}}{2}$, and $p_{3}(\mathbf{b})=b_{(4)}$. Observe that $\mathbb{G}$ is a GLP position auction and that $\mathbb{G}$ is not monotone.

We next show that $\mathbb{G}$ is a VCG cover. Let $\mathbf{v} \in V$ be an arbitrary valuation vector. We need to find a bid profile $\mathbf{b}(\mathbf{v})$ such that $\psi^{\mathbb{G}}(\mathbf{b}(\mathbf{v}))=\varphi^{v c g}(\mathbf{v})$. Note that $p_{1}^{v c g}(\mathbf{v})=\frac{90 v_{(2)}+9 v_{(3)}+v_{(4)}}{100}, p_{2}^{v c g}(\mathbf{v})=\frac{9 v_{(3)}+v_{(4)}}{10}$ and $p_{3}^{v c g}(\mathbf{v})=v_{(4)}$. We define the bid profile $\mathbf{b}(\mathbf{v})$ recursively: $b_{\delta(\mathbf{v}, 4)}(\mathbf{v})=p_{3}^{v c g}(\mathbf{v}), b_{\delta(\mathbf{v}, 3)}(\mathbf{v})=2 p_{2}^{v c g}(\mathbf{v})-b_{\delta(\mathbf{v}, 4)}(\mathbf{v}), b_{\delta(\mathbf{v}, 2)}(\mathbf{v})=b_{\delta(\mathbf{v}, 3)}+p_{1}^{v c g}(\mathbf{v})$ and $b_{\delta(\mathbf{v}, 1)}(\mathbf{v})=$ $b_{\delta(\mathbf{v}, 2)}(\mathbf{v})+1$.

By Lemma 3 and by the construction of $\mathbf{b}(\mathbf{v}), s(\mathbf{b}(\mathbf{v}), i)=s(\mathbf{v}, i)$ for $i=1, \ldots, 4$. In addition, observe that $p_{j}(\mathbf{b}(\mathbf{v}))=$ $p_{j}^{v c g}(\mathbf{v})$ for $j=1,2,3$. Therefore, $\psi^{\mathbb{G}}(\mathbf{b}(\mathbf{v}))=\varphi^{v c g}(\mathbf{v})$, i.e., $\mathbb{G}$ is a VCG cover. 
Suppose in negation that there exists an individually rational mediator $\mathbf{m}$ that implements the VCG outcome function in $\mathbb{G}$. Consider the following vector of valuations $\mathbf{v}=(14,12,14,1)$. Suppose players 1,3 and 4 use the $T$-strategy. We show that player 2 is better off participating directly in the auction, which contradicts our assumption that $\mathbf{m}$ implements the VCG outcome function in $\mathbb{G}$. If player 2 (with valuation 12 ) uses the $T$-strategy, she obtains position 3 and pays 1 , and therefore her utility is $1(12-1)=11$. Suppose player 2 participates directly in the auction. Let $b_{i}, i=1,2,3,4$, be the bids submitted to the auction ( $b_{i}$, for $i=1,3,4$, are the bids submitted by the mediator $\mathbf{m}$ ). If $b_{i}=0$ for some $i \in\{1,3,4\}$, then by bidding $b_{2}=0.5$, player 2 gains at least position 3 , pays at most 0.5 , and therefore strictly improves her utility, yielding a contradiction. Suppose $b_{i}>0$ for every $i \in\{1,3,4\}$. We distinguish between the following cases, while in each case the idea is to derive constraints about the possible bids $\mathbf{m}$ can submit on behalf of at least one of the players that use the $T$-strategy, and show that under these constraints player 2 has a beneficial deviation:

1. $b_{1} \geqslant b_{3} \geqslant b_{4}$.

We first show that $b_{4} \leqslant 1$. Indeed, if $b_{4}>1$, by bidding $b_{2}=\frac{b_{4}+1}{2}, q_{4}(\mathbf{b})>1$, which contradicts individual rationality. If $b_{4}<1$, by letting $b_{2}=b_{4}, q_{2}(\mathbf{b})<1$, and since $s(\mathbf{b}, 2) \in K$, player 2 's utility is at least $1\left(12-q_{2}(\mathbf{b})\right)>11$. Suppose $b_{4}=1$. If $b_{2}<b_{4}, q_{1}(\mathbf{b})=b_{3}-b_{4}$. Since $\mathbf{m}$ is individually rational, $b_{3}-b_{4} \leqslant 14$. Hence, $b_{3} \leqslant 15$. Let $b_{2}=b_{3}$. Therefore, by our standard priority rule, $s(\mathbf{b}, 2)=2$; hence, $q_{2}(\mathbf{b}) \leqslant \frac{15+1}{2}$. Therefore, player 2's utility is at least $10(12-8)>11$.

2. $b_{1} \geqslant b_{4}>b_{3}$.

We first show that $b_{3} \leqslant 1$. Indeed, if $b_{3}>1$, by letting $b_{2}=1$ we have $q_{4}(\mathbf{b})=\frac{b_{3}+b_{2}}{2}>1$, contradicting individual rationality. If $b_{2}<b_{3}, q_{1}(\mathbf{b})=b_{4}-b_{3}$, and therefore, $b_{4}-b_{3} \leqslant 14$ by individual rationality. Hence, $b_{4} \leqslant 15$. Let $b_{2}=b_{4}$. By the priority rule, $s(\mathbf{b}, 2)=2$; hence, $q_{2}(\mathbf{b}) \leqslant \frac{15+1}{2}$. Therefore player 2's utility is at least $10(12-8)>11$.

3. $b_{4}>b_{1} \geqslant b_{3}$.

We first show that $b_{3} \leqslant 1$. Indeed, if $b_{3}>1$, by letting $b_{2}>b_{4}, s(\mathbf{b}, 4)=2$; therefore $q_{4}(\mathbf{b})=\frac{b_{1}+b_{3}}{2}>1$, which contradicts individual rationality. Suppose $b_{2}<b_{3}$. Therefore $q_{4}(\mathbf{b})=b_{1}-b_{3}$, and by individual rationality $b_{1} \leqslant 2$. Let $b_{2}=3$. By the priority rule, $s(\mathbf{b}, 2) \in\{1,2\}$; since $q_{2}(\mathbf{b}) \leqslant 3$, player 2 's utility is at least $10(12-3)>11$.

4. $b_{3}>b_{1} \geqslant b_{4}$.

By the arguments we use at the first case and because of the symmetry of players 1 and 3 , we obtain that $b_{1} \leqslant$ 15 and $b_{4} \leqslant 1$. Let $b_{2}=\frac{b_{3}+b_{1}}{2}$. Hence $s(\mathbf{b}, 2)=2$; therefore $q_{2}(\mathbf{b}) \leqslant \frac{15+1}{2}=8$. Therefore, player 2's utility is at least $10(12-8)>12$.

The cases $b_{3} \geqslant b_{4}>b_{1}$ and $b_{4}>b_{3} \geqslant b_{1}$ are similar to cases 2 and 3 , respectively. We obtained that player 2 benefits from participating directly in the auction, which contradicts our assumption that $\mathbf{m}$ is an individually rational mediator, which implements $\varphi^{v c g}$ in $\mathbb{G}$.

To summarize, we give a minimal set of conditions, which is sufficient for transforming a large class of position auctions to the VCG position auction by truthful and individual rational mediation.

\subsection{VCG cover}

Conditions 1 and 3 in Theorem 4 are easy to verify. However verifying condition 2 is more challenging. In Theorem 5 below we derive three conditions, which together are sufficient for a GLP position auction to be a VCG cover. We first need a few notations. Let $\mathbf{Z}=\left\{\left(z_{1}, \ldots, z_{n}\right): z_{1} \geqslant z_{2} \geqslant \cdots \geqslant z_{n} \geqslant 0\right\}$ be the set of all ordered vectors of bids. Let $\mathbf{G}$ be a position auction, and let $p_{j}$ be the position payment function in $\mathbb{G}$ for every $j \in K$. Since $p_{j}$ is anonymous, there exists a real valued function $\tilde{p}_{j}: \mathbf{Z} \rightarrow \mathbb{R}_{+}$such that $p_{j}(\mathbf{b})=\tilde{p}_{j}\left(\mathbf{b}^{*}\right)$ for every bid profile $\mathbf{b}$, where $\mathbf{b}^{*}=\left(b_{(1)}, b_{(2)}, \ldots, b_{(n)}\right)$. Accordingly, we define $\tilde{p}_{m+1}$ to be identically zero.

For every $1 \leqslant j \leqslant m+1$, let

$$
T(j)=\left\{k \in N \mid \tilde{p}_{j}(\mathbf{z})=\tilde{p}_{j}(\hat{\mathbf{z}}) \forall \mathbf{z}, \hat{\mathbf{z}} \in \mathbf{Z} \text { for which } \mathbf{z}_{-k}=\hat{\mathbf{z}}_{-k}\right\},
$$

and let $D(j)=N \backslash T(j)$. Note that $T(m+1)=N$ and therefore $D(m+1)=\emptyset$.

$D(j)$ is the set of coordinates on which $\tilde{p}_{j}$ depends. For example, in the next-price auction $D(j)=\{j+1\}$, and in the VCG position auction $D(j)=\{j+1, j+2, \ldots, m+1\}$. For every finite set of integers, $S \subseteq\{1, \ldots, n\}$, let $\mathbf{I}(S)$ denote the smallest integers in $S$. For $S=\emptyset$ we define $\mathbf{l}(S)=n+1$. The proof of the following theorem is given in Section 8 .

Theorem 5. Let $\mathbb{G}=\mathbb{G}(\alpha, \mathbf{p})$ be a GLP position auction. $\mathbb{G}$ is a VCG cover if the following three conditions hold:

1. For every $\mathbf{z} \in \mathbf{Z}$, for every $j \in K$, and for every $c \in\left[\tilde{p}_{j+1}(\mathbf{z}), \infty\right)$ there exists $\mathbf{z}^{\prime} \in \mathbf{Z}$ with $\tilde{p}_{j}\left(\mathbf{z}^{\prime}\right)=c$, such that $z_{k}^{\prime}=z_{k}$ for all $k \geqslant \mathbf{l}(D(j+1))$.

2. For every $j \in K, \tilde{p}_{j}()$ is a continuous function.

3. For every $j \in K, \tilde{p}_{j}()$ is monotone increasing in $z_{k}$ for every $k \in D(j) \backslash \bigcup_{t=j+1}^{m+1} D(t)$. 


\section{Remarks.}

1. The first condition in Theorem 5 roughly states as follows: If in a certain ordered bid profile the player in position $j+1$ pays $\hat{c}$, then for every $c \geqslant \hat{c}$ there exist an ordered bid profile in which the player in position $j$ pays $c$, and the payments of the players in positions $j+1, j+2, \ldots$ are not changed.

2. Theorem 5 can be used to indirectly prove that the position auction in Example 3 is a VCG cover.

The proof of Theorem 5 is given in Section 8. In the following examples we apply Theorems 4 and 5 to interesting classes of position auctions, generalized next-price position auctions and weighted next-price position auctions, which are defined bellow.

Definition 9 (Generalized next-price position auctions). A position auction $\mathbb{G}(\alpha, \mathbf{p})$ is called a generalized next-price position auction if there exists an integer-valued function, $d$ defined on $K$ such that the following holds:

$$
p_{j}(\mathbf{b})=b_{(d(j))} \text { for every } \mathbf{b} \in B,
$$

where $b_{(s)}=0$ for every $s>n$.

Generalized next-price position auctions are not used in practice these days, but at least in the one-position case they were recommended to sellers in certain circumstances. For example, see Monderer and Tennenholtz (2004b), where it is written: "Our recommendation to organizers of auctions is to conduct k-price auctions, $k \geqslant 3$ in environments in which buyers are risk-seeking. The recommendation is given both for, a setup in which each organizer is a monopolist, and for one of oligopolistic competition." See also Tauman (2002), where it is proved that a third-price auction yields a higher revenue than secondprice and first-price auctions in a model in which every participants know all valuations, but the organizer does not know the participants' valuations; in Tauman's model, the risk attitude of the buyers is not restricted.

Theorem 6. Let $\mathbb{G}$ be a generalized next-price position auction, and let d be an integer-valued function defined on $K$, which satisfies (4). There exists an individually rational mediator that implements $\varphi^{v c g}$ in $\mathbb{G}$ if and only if the following two conditions hold:

(i) $d(j+1)>d(j)$ for $j=1, \ldots, m-1$;

(ii) $d(m) \leqslant n$.

The proof of Theorem 6 is given in Section 8. The main ideas in the proof are as follows: Suppose there exists an individually rational mediator that implements $\varphi^{v c g}$ in $\mathbb{G}$. Fix some position $j \in K$. Since $p_{j}^{v c g}(\mathbf{v}) \geqslant p_{j+1}^{v c g}(\mathbf{v})$ for every $\mathbf{v} \in \mathbf{V}$, $d(j+1) \geqslant d(j)$ for every $1 \leqslant j \leqslant m-1$. Since there exists $\mathbf{v}$ for which $p_{j}^{v c g}(\mathbf{v})>p_{j+1}^{v c g}(\mathbf{v}), d(j+1)>d(j)$. This proves that (i) holds. To prove that (ii) holds, note that there exists $\mathbf{v} \in \mathbf{V}$ for which the player that obtains position $m$ has a positive VCG payment. Therefore, $d(m) \leqslant n$ because otherwise, $p_{m}$ will be identically zero.

We also have to show that conditions (i) and (ii) are sufficient. Indeed, suppose these conditions are satisfied. We have to prove that there exists an individually rational mediator that implements $\varphi^{v c g}$ in $\mathbb{G}$. By Theorem 4 it suffices to prove that $\mathbb{G}$ is a monotone GLP position auction, which is also a VCG cover; the non-trivial task is to show that $\mathbb{G}$ is a VCG cover, and we use Theorem 5 to show it. That is, we have to prove that the three conditions in Theorem 5 are satisfied. However, only the first condition is not obvious. In order to prove that the first condition in Theorem 5 holds, fix some $\mathbf{z} \in \mathbf{Z}$ and some $c \in\left[\tilde{p}_{j+1}(\mathbf{b}), \infty\right)$. Note that $D(j)=\{d(j)\}$. Let $\mathbf{z}^{\prime}$ be the profile obtained from $\mathbf{z}$ by replacing $z_{k}$ by $c$ for every $k<d(j+1)$. Since $d(j)<d(j+1)$ we obtain that $\tilde{p}_{j}\left(\mathbf{z}^{\prime}\right)=c$.

Definition 10 (Weighted next-price position auctions). A position auction $\mathbb{G}(\alpha, \mathbf{p})$ is called a weighted next-price position auction if there exist constants $c_{j}, 1 \leqslant j \leqslant m$, with $c_{j} \geqslant 1$, such that the payment scheme $\mathbf{p}$ has the following form: For every $j \in K$ and for every $\mathbf{b} \in B, p_{j}(\mathbf{b})=\frac{b_{(j+1)}}{c_{j}}$.

The proof of the following theorem, which is similar to the proof of Theorem 6, appears in Section 8 .

Theorem 7. Let $\mathbb{G}$ be a weighted next-price position auction with the weights $c_{1}, c_{2}, \ldots, c_{m}$. There exists an individually rational mediator that implements $\varphi^{v c g}$ in $\mathbb{G}$ if and only if $c_{1} \geqslant \cdots \geqslant c_{m}$.

\section{Self-price position auctions}

Let $\mathbb{G}$ be a self-price position auction as described in Section 2. In Example 1 we showed that when there are one position and two players, the VCG outcome function is implemented in $\mathbb{G}$ by an individually rational mediator. The proof we gave in this example can be easily generalized to show that the VCG outcome function can be implemented by an individually rational mediator in a self-price position auction in which there is one position and an arbitrary number of 
players, $n \geqslant 2$. Next we show that it is impossible to implement the VCG outcome function, even by a non-individually rational mediator, in a self-price position auction, which has more than one position $(m>1)$.

Theorem 8. Let $\mathbb{G}$ be a self-price position auction with more than one position. There does not exist a mediator that implements the VCG outcome function in $\mathbb{G}$.

Proof. Let $\mathbf{v} \in V$ be the following valuation profile: $v_{n}=10$ and $v_{1}=v_{2}=\cdots=v_{n-1}=5$. The VCG position auction assigns position 1 to player $n$ and position 2 to player 1, and each of these players pays 5 . In order to implement such an outcome, a mediator must bid 5 on behalf of player $n$, so that player $n$ will pay 5 , and it must bid less than 5 on behalf of any other player, otherwise, by the priority rule, player $n$ will not get position 1 . In particular, even if player 1 is assigned to position 2, she will pay less than 5 . Hence, no mediator can implement the VCG outcome function in $\mathbb{G}$.

The impossibility result in Theorem 8 follows from the ties, and it can be proved for every random priority rule. In contrast, we give below a positive result for self-price position auctions with the first arrival rule-in such auctions ties are impossible.

As discussed in Section 3.1, the fixed and random priority rules are just convenient ways to model the first-arrival rule, which is common in practice.

When one attempts to directly model position auctions that use the first-arrival rule without these modeling choices he tackles a lot of modeling problems. In particular, it is not clear how to model a position auction with the first-arrival rule as a game with incomplete information. To do this, one has to allow a player not only to submit a bid but also to decide about the time of the bid. This raises a lot of additional modeling problems, such as determining the relationship between the time a player decides to submit a bid and the time in which this bid is actually recorded. Nevertheless, we next analyze mediators in position auctions, which use the first-arrival rule. We will define ex post equilibrium and the notion of implementation by truthful mediation without explicitly modeling well-defined games. We will show that in this case there is a way to implement the VCG outcome function in a self-price position auction. In particular, we will find an individually rational mediator that does the job. Let $\mathbb{G}$ be a position auction with the first-arrival rule. Every mediator for $\mathbb{G}$ has the ability to determine the order in which the bids he submits on behalf of the players are recorded; he can just submit the bids sequentially, waiting for a confirmation before submitting the next bid. We need the following notations.

Every order of bidding can be described by some $\gamma \in \Gamma$; $i$ bids before $k$ if and only if $\gamma_{i}<\gamma_{k}$. Hence, an order of bids can serve as a priority rule. For every order of bids $\gamma$ and a vector of bids $\mathbf{b}$ we define $s(\mathbf{b}, \gamma, i)$ as the position assigned to $i$. We denote the payment of $i$ when the vector of bids is $\mathbf{b}$ and the order of bidding is $\gamma$ by $q_{i}(\mathbf{b}, \gamma)=p_{s(\mathbf{b}, \gamma, i)}(b)$, and we denote $u_{i}\left(v_{i}, \mathbf{b}, \gamma\right)$ the utility of $i$.

A mediator for $\mathbb{G}$ should determine the bids of the players who use its services and also the order of bids as a function of the reported types. However, all mediators discussed in this section will use the same rule to determine the order of bids: If all players report the vector of types $\hat{\mathbf{v}}$, the mediator uses the order of bids $\gamma^{\hat{\mathbf{v}}}$, which is defined as follows: $\gamma_{i}^{\hat{\mathbf{v}}}<\gamma_{k}^{\hat{\mathbf{v}}}$ if and only if $\hat{\mathbf{v}}_{i}>\hat{\mathbf{v}}_{k}$, or $\hat{\mathbf{v}}_{i}=\hat{\mathbf{v}}_{k}$ and $i<k$. For example, if $n=3$ and the reported types are $\hat{\mathbf{v}}=(6,7,6), \gamma^{\hat{\mathbf{v}}}=(2,1,3)$. If only a strict subset of the players use the mediator's services, the mediator applies the same order of bids rule to this subset. A mediator for a position auction with the first arrival rule is therefore defined by a vector $\mathbf{m}=\left(\mathbf{m}_{S}\right)_{S \subseteq N}$. However, such a mediator is called a scheduling mediator in order to stress the fact that it determines not only the bids but also the order of bids. To summarize: If all players use the scheduling mediator $\mathbf{m}$, and the reported bids are $\hat{\mathbf{v}}$, the scheduling mediator bids $\mathbf{m}_{N}(\hat{\mathbf{v}})_{i}$ on behalf of $i, i$ receives the position $s\left(\hat{\mathbf{v}}, \gamma^{\hat{\mathbf{v}}}, i\right)$, and pays $q_{i}\left(m_{N}(\hat{\mathbf{v}}), \gamma^{\hat{\mathbf{v}}}\right)$. If only the subset $S$ uses the mediator's services, the reported types are $\hat{\mathbf{v}}_{S}$ and the other players bid directly $\mathbf{b}_{-S}$, the actual order of bids is not uniquely determined. If this order is $\gamma$, the position of $i \in N$ is $s(\mathbf{b}, \gamma, i)$, and its payment is $q_{i}(\mathbf{b}, \gamma)$, where $\mathbf{b}=\left(\mathbf{m}_{S}\left(\hat{\mathbf{v}}_{S}\right), \mathbf{b}_{-S}\right)$. In particular, if every player is using the $T$-strategy and the players' profile of types is $\mathbf{v}$, the outcome generated by the scheduling mediator is

$$
\psi^{\mathbf{m}}(\mathbf{v})=\left(\mathbf{s}\left(\mathbf{v}, \gamma^{\mathbf{v}}\right), \mathbf{q}\left(\mathbf{m}_{N}(\mathbf{v}), \gamma^{\mathbf{v}}\right)\right)
$$

But why should the players use the $T$-strategy? Assume all players but $i$ use the $T$-strategy. If player $i$ deviates from the $T$-strategy by reporting a false type to the scheduling mediator, the resulting outcome is well defined. On the other hand, when this player sends a bid directly to the auctioneer, the resulting outcome is not clear because the order of bids is not clear. ${ }^{21}$ A desired scheduling mediator would be one for which no player would want to deviate from the $T$-strategy independently of the order in which the bids are recorded because of his deviation. More precisely:

Definition 11. Let $\mathbb{G}$ be a position auction with the first-arrival rule, and let $\mathbf{m}$ be a scheduling mediator for $\mathbb{G}$. The $T$ strategy profile is an ex post equilibrium with respect to $\mathbf{m}$ if for every player $i$, for every type $v_{i}$, and for every vector of types of the other players, $\mathbf{v}_{-i}$, the following two conditions hold:

\footnotetext{
21 It is clear however, that the resulting order $\gamma$ is consistent with the well-defined order of bids of $N \backslash\{i\}$.
} 
F1: $i$ is not better off when he gives the scheduling mediator the right to play and reports a false type. That is, for every $\hat{v}_{i} \in V_{i}$

$$
u_{i}\left(v_{i}, \mathbf{m}_{N}\left(v_{i}, \mathbf{v}_{-i}\right), \gamma^{\left(v_{i}, v_{-i}\right)}\right) \geqslant u_{i}\left(v_{i}, \mathbf{m}_{N}\left(\hat{v}_{i}, \mathbf{v}_{-i}\right), \gamma^{\left(\hat{v}_{i}, v_{-i}\right)}\right) .
$$

F2: $i$ is not better off when he bids directly, independently of the resulting order of recorded bids. That is, for every $b_{i} \in B_{i}$, and for every $\gamma \in \Gamma$, which is consistent with the order of bids of members of $N \backslash\{i\}$ resulting from the vector of types $v_{-i}$,

$$
u_{i}\left(v_{i}, \mathbf{m}_{N}\left(v_{i}, \mathbf{v}_{-i}\right), \gamma^{\left(v_{i}, \mathbf{v}_{-i}\right)}\right) \geqslant u_{i}\left(v_{i}, b_{i}, \mathbf{m}_{N \backslash\{i\}}\left(\mathbf{v}_{-i}\right), \gamma\right) .
$$

The notion of individually rational scheduling mediators is analogously defined, i.e. the mediator guarantees a nonnegative utility for every player that uses the $T$-strategy. Formally,

Definition 12. Let $\mathbb{G}$ be a position auction with the first-arrival rule. A scheduling mediator $\mathbf{m}$ for $\mathbb{G}$ is individually rational, if for every player $i$, for every subset $S \subseteq N$ such that $i \in S$, for every $\mathbf{v}_{S}$, and for every $\mathbf{b}_{-S}, u_{i}\left(v_{i}, \mathbf{m}_{S}\left(\mathbf{v}_{S}\right), \mathbf{b}_{-S}, \gamma\right) \geqslant 0$ for every $\gamma \in \Gamma$, which is consistent with the standard order of bids of $S$ determined by the mediator when the reported types are $\mathbf{v}_{S}$.

The notion of implementation by truthful mediation remains as before: The scheduling mediator $\mathbf{m}$ implements the VCG outcome function in $\mathbb{G}$ if $\psi^{\mathbf{m}}=\varphi^{v c g}$.

Our previous results remain true for scheduling mediators for position auctions with the first arrival rule. Next we show that in contrast to Theorem 8, it is possible to implement the VCG outcome function in every self-price position auction with the first-arrival rule.

Theorem 9. Let $\mathbb{G}=\mathbb{G}(\alpha, \mathbf{p})$ be the self-price position auction with the first arrival rule. There exists an individually rational scheduling mediator that implements the VCG outcome function in $\mathbb{G}$.

Proof. We define a scheduling mediator $\mathbf{m}$ as follows: For every $\mathbf{v} \in V, \mathbf{m}_{N}(\mathbf{v})=\mathbf{b}(\mathbf{v})$, where $\mathbf{b}(\mathbf{v})$ is the bid profile defined as follows: $\mathbf{b}_{i}(\mathbf{v})=p_{s\left(\mathbf{v}, \gamma^{\mathbf{v}}, i\right)}^{v c g}(\mathbf{v})$ for every $i$ such that $1 \leqslant s\left(\mathbf{v}, \gamma^{\mathbf{v}}, i\right) \leqslant m$, and $\mathbf{b}_{i}(\mathbf{v})=\frac{p_{s\left(\mathbf{v}, \gamma^{\mathbf{v}}, m\right)}^{v c g}(\mathbf{v})}{2}$ for every $i$ such that $s\left(\mathbf{v}, \gamma^{\mathbf{v}}, i\right)=m+1$. For every strict subset, $S \subset N, \mathbf{m}_{S}\left(\mathbf{v}_{S}\right)=\mathbf{v}_{S}$. Since the order of bids is determined by $\mathbf{v}$ and not by the bids themselves, $\psi^{\mathbf{m}}(\mathbf{v})=\varphi^{v c g}(\mathbf{v})$ for every $\mathbf{v} \in V$. We next show that the $T$-strategy is an ex post equilibrium. Suppose all players but $i$ use the $T$-strategy. Since telling the truth is a dominant strategy in the VCG position auction with the fixed priority rule, it is not beneficial for player $i$ to report a false value to the mediator. Suppose player $i$ bids directly in the auction. Let $v_{-i}$ be the values of all other players. Let $b_{i}$ be player $i$ 's bid, and let $\tilde{b}=\left(b_{i}, v_{-i}\right)$. Let $\tilde{\gamma} \in \Gamma$ be a priority order consistent with order of bids sent by the scheduling mediator $\mathbf{m}$. Let $j=s(\tilde{b}, \tilde{\gamma}, i)$. Without loss of generality, we assume that $j \in K$. Since the mediator bids on behalf of all other players their reported values, player $i$ will pay at least $v_{(j+1)}$. If player $i$ would have deviated to position $j$ in the VCG position auction with the fixed order $\tilde{\gamma}$, then by part 1 of Lemma 3 , her payment would have been at least $v_{(j+1)}$. Therefore direct participation in the position auction is not beneficial for $i$.

The mediator is individually rational since $p_{j}^{v c g}(\mathbf{b}) \leqslant b_{(j)}$ for every $j$ and every bid profile $\mathbf{b}$.

\section{Position auctions with quality factors}

Every position auction is player-anonymous except for ties. However, companies like Google express preferences over players by introducing quality factors (see e.g., Varian, 2007); player $i$ has a fixed quality factor $\beta_{i}>0$. Let $\mathbb{G}=\mathbb{G}(\alpha, \mathbf{p})$ be a position auction, and let $\beta=\left(\beta_{1}, \ldots, \beta_{n}\right)$ be a vector of quality factors. We define a new auction, $\mathbb{G}(\beta, \alpha, \mathbf{p})$, as follows: If the players send the vector of bids $\mathbf{b}=\left(b_{1}, b_{2}, \ldots, b_{n}\right)$ the auctioneer chooses the allocation $\mathbf{s}_{\beta}(\mathbf{b})=\mathbf{s}(\beta \mathbf{b})$ where $\beta \mathbf{b}=\left(\beta_{1} b_{1}, \beta_{2} b_{2}, \ldots, \beta_{n} b_{n}\right)$. If $i$ receives position $j$, that is $s(\beta \mathbf{b}, i)=j, i$ pays $\frac{1}{\beta_{i}} p_{j}(\beta \mathbf{b})$ per click. Every such auction, $\mathbb{G}(\beta, \alpha, p)$, is called a $\beta$-position auction. The $\beta$-VCG position auction is $\mathbb{G}\left(\beta, \alpha, \mathbf{p}^{v c g}\right)$. It chooses the allocation of the weighted VCG mechanism (see e.g., Roberts, 1979) with the vector of weights $\beta$, and the payment of a player equals the standard weighted VCG payment. As observed by Varian (2007), every position auction with quality factors can be reformulated as a position auction without quality factors by redefining bidders' valuations to be the product of their original valuations and their quality factors, i.e. $v_{i} \beta_{i}$ is considered to be player $i$ 's valuation. Based on this the following observation holds:

Observation 10. Let $\mathbb{G}=\mathbb{G}(\alpha, \mathbf{p})$ be a position auction, and let $\beta=\left(\beta_{1}, \beta_{2}, \ldots, \beta_{n}\right)$ be a vector of quality factors. There exists an individually rational mediator that implements the $\beta$-VCG outcome function in $\mathbb{G}(\beta, \alpha, \mathbf{p})$ if and only if there exists an individually rational mediator that implements the VCG outcome function in $\mathbb{G}(\alpha, \mathbf{p})$.

Hence, the $\beta$ versions of all the theorems proved in previous sections hold. 


\section{The remaining proofs}

Proof of Lemma 3. ${ }^{22}$ 1. Let $j \in K$. Note that by the VCG payment scheme (3), $p_{j}^{v c g}(\mathbf{b})$ is a convex combination of $b_{(j+1)}, b_{(j+2)}, \ldots, b_{(m+1)}$. Therefore, it never exceeds the maximal element in the sequence, $b_{(j+1)}$.

2. If $j=m$ then for every $\mathbf{b} \in B$

$$
p_{j}^{v c g}(\mathbf{b})=b_{(j+1)} \geqslant 0=p_{j+1}^{v c g}(\mathbf{b}) .
$$

Suppose $j<m$. Hence, $j+1 \in K$. Since $b_{(j+1)} \geqslant b_{(j+2)}$,

$$
p_{j}^{v c g}(\mathbf{b}) \geqslant \frac{b_{(j+2)}\left(\alpha_{j}-\alpha_{j+1}\right)}{\alpha_{j}}+\frac{\sum_{k=j+2}^{m+1} b_{(k)}\left(\alpha_{k-1}-\alpha_{k}\right)}{\alpha_{j}} .
$$

Note that the right-hand side of (5) equals to

$$
b_{(j+2)}-\sum_{k=j+2}^{m} \frac{\alpha_{k}}{\alpha_{j}}\left(b_{(k)}-b_{(k+1)}\right) .
$$

Since $\alpha_{j}>\alpha_{j+1}$,

$$
p_{j}^{v c g}(\mathbf{b}) \geqslant b_{(j+2)}-\sum_{k=j+2}^{m} \frac{\alpha_{k}}{\alpha_{j+1}}\left(b_{(k)}-b_{(k+1)}\right)=p_{j+1}^{v c g}(\mathbf{b}) .
$$

Obviously if $b_{(j+1)}=b_{(j+2)}=\cdots=b_{(m+1)}$ then $p_{j}^{v c g}(\mathbf{b})=p_{j+1}^{v c g}(\mathbf{b})$. If $b_{(j+1)}=b_{(j+2)}=\cdots=b_{(m+1)}$ doesn't hold, it implies that there is a strong inequality in either (5) or (6). This implies that $p_{j}^{v c g}(\mathbf{b})>p_{j+1}^{v c g}(\mathbf{b})$.

Proof of Theorem 4 (Part (i)). Let $\mathbb{G}=\mathbb{G}(\alpha, \mathbf{p})$ be a monotone GLP position auction, which is also a VCG cover. We show that Mediator 2 below is an individually rational mediator that implements the VCG outcome function in $\mathbb{G}$.

\section{Mediator 2.}

(a) $\mathbf{m}_{N}(\mathbf{v})=\mathbf{b}(\mathbf{v})$ for every $\mathbf{v} \in V$, where $\mathbf{b}(\mathbf{v})$ is some bid profile such that $\psi^{\mathbb{G}}(\mathbf{b}(\mathbf{v}))=\varphi^{v c g}(\mathbf{v})$. Since $\mathbb{G}$ is a VCG cover, such a function $\mathbf{b}$ exists.

(b) Let $\epsilon>0$ be fixed. For every $i$ and for every $\mathbf{v}_{-i} \in \mathbf{V}_{-i}$, let $\mathbf{v}^{i}=\left(\mathbf{v}_{-i}, M\left(\mathbf{v}_{-i}\right)\right)$, where $M\left(\mathbf{v}_{-i}\right)=\epsilon+\max _{j \neq i} v_{j}$. $\mathbf{m}_{N \backslash\{i\}}\left(\mathbf{v}_{-i}\right)=\mathbf{b}_{-i}\left(\mathbf{v}^{i}\right)$ for every $i \in N$ and every $\mathbf{v}_{-i} \in \mathbf{V}_{-i}$.

(c) For every $S \subseteq N$ such that $1 \leqslant|S| \leqslant n-2, \mathbf{m}_{S}\left(\mathbf{v}_{S}\right)=\mathbf{v}_{S}$ for every $\mathbf{v}_{S} \in \mathbf{V}_{S}$.

Let $\mathbf{m}$ be Mediator 2 as defined above. We have to show that $\mathbf{m}$ implements $\varphi^{v c g}$ in $\mathbb{G}$. By (a), $\varphi^{\mathbf{m}}=\varphi^{v c g}$. We proceed to show that the $T$-strategy profile is an ex post equilibrium. Suppose all players but $i$ are using the $T$-strategy. By the truthfulness property of the VCG position auction, it is not beneficial for player $i$ to report a false value. Next we show that it is not beneficial for player $i$ to participate in the auction directly. Suppose player $i$ bids $b_{i}$ directly in the auction. Let $\tilde{\mathbf{b}}=\left(\mathbf{b}_{-i}\left(\mathbf{v}^{i}\right), b_{i}\right)$ be the profile of bids reported to the auctioneer, and let $j=s(\tilde{\mathbf{b}}, i)$ be the position of $i$ resulting from her deviation. Let $h=s(\mathbf{b}(\mathbf{v}), i)$ be $i$ 's position if $i$ uses the $T$-strategy. We have to show that

$$
\alpha_{h}\left(v_{i}-p_{h}(\mathbf{b}(\mathbf{v}))\right) \geqslant \alpha_{j}\left(v_{i}-p_{j}(\tilde{\mathbf{b}})\right) .
$$

Because $\varphi^{\mathbf{m}}=\varphi^{v c g}$, it suffices to show that:

$$
\alpha_{h}\left(v_{i}-p_{h}^{v c g}(\mathbf{v})\right) \geqslant \alpha_{j}\left(v_{i}-p_{j}(\tilde{\mathbf{b}})\right) .
$$

Since in the VCG position auction the utility of a truth-telling player is always non-negative, inequality (7) holds trivially if $\alpha_{j}=0$. In particular, it holds if $j=-1$ or $j=m+1$, and therefore, we can assume without loss of generality that $1 \leqslant j \leqslant m$. Let $b_{i}^{\prime} \in \mathbf{B}_{i}$ be some bid for player $i$ such that $b_{i}^{\prime}=\left(\mathbf{v}_{-i}, b_{i}^{\prime}\right)_{(j)}$. Before we continue we need the following discussion: The allocation rule in the VCG auction is defined by the priority rule $\tilde{\gamma}=(1, \ldots, n) .{ }^{23}$ Let $\operatorname{VCG}(\gamma)$ be the VCG position auction, in which the priority rule is $\gamma$. The position payment scheme in $\operatorname{VCG}(\gamma)$ is the same as in $\operatorname{VCG}(\tilde{\gamma})$. We define $s(\mathbf{b}, i, \gamma)$ to be player $i$ 's position, when the bid profile is $\mathbf{b}$, and the priority rule is $\gamma$. Note that $s(\mathbf{b}, i, \tilde{\gamma})$ is just $s(\mathbf{b}, i)$.

22 The proof of some parts of Lemma 3 can also be deduced from a recursive definition of the VCG payments given in Varian (2007) and in Edelman et al. (2007).

23 See Section 3.1 for the discussion of priority rules. 
Since $b_{i}^{\prime}=\left(\mathbf{v}_{-i}, b_{i}^{\prime}\right)_{(j)}$, there exists $\gamma \in \Gamma$ such that $s\left(\left(\mathbf{v}_{-i}, b_{i}^{\prime}\right), i, \gamma\right)=j$. Let $\hat{h}=s(\mathbf{v}, i, \gamma)$. Observe that $v_{(\hat{h})}=v_{(h)}$. Since players with identical values, which report truthfully, have the same utility in the VCG position auction,

$$
\alpha_{h}\left(v_{(h)}-p_{h}^{v c g}(\mathbf{v})\right)=\alpha_{\hat{h}}\left(v_{(\hat{h})}-p_{\hat{h}}^{v c g}(\mathbf{v})\right) .
$$

Since $v_{(h)}=v_{(\hat{h})}=v_{i}$,

$$
\alpha_{h}\left(v_{i}-p_{h}^{v c g}(\mathbf{v})\right)=\alpha_{\hat{h}}\left(v_{i}-p_{\hat{h}}^{v c g}(\mathbf{v})\right) .
$$

Since the payment schemes in VCG and VCG $(\gamma)$ are identical,

$$
\alpha_{\hat{h}}\left(v_{i}-p_{\hat{h}}^{v c g}(\mathbf{v})\right)=\alpha_{\hat{h}}\left(v_{i}-p_{\hat{h}}^{V C G(\gamma)}(\mathbf{v})\right) .
$$

Because of the truth-telling property of $V C G(\gamma)$

$$
\alpha_{\hat{h}}\left(v_{i}-p_{\hat{h}}^{V C G(\gamma)}(\mathbf{v})\right) \geqslant \alpha_{j}\left(v_{i}-p_{j}^{V C G(\gamma)}\left(\mathbf{v}_{-i}, b_{i}^{\prime}\right)\right) .
$$

Therefore

$$
\alpha_{h}\left(v_{i}-p_{h}^{v c g}(\mathbf{v})\right) \geqslant \alpha_{j}\left(v_{i}-p_{j}^{v c g}\left(\mathbf{v}_{-i}, b_{i}^{\prime}\right)\right) .
$$

Since the VCG position auction is a GLP position auction, and $\left(v_{-i}, b_{i}^{\prime}\right)_{(l)}=v_{(l)}^{i}$ for every $l>j$,

$$
\alpha_{j}\left(v_{i}-p_{j}^{v c g}\left(\mathbf{v}_{-i}, b_{i}^{\prime}\right)\right)=\alpha_{j}\left(v_{i}-p_{j}^{v c g}\left(\mathbf{v}^{i}\right)\right) .
$$

By the definition of $\mathbf{b}\left(\mathbf{v}^{i}\right)$,

$$
\alpha_{j}\left(v_{i}-p_{j}^{v c g}\left(\mathbf{v}^{i}\right)\right)=\alpha_{j}\left(v_{i}-p_{j}\left(\mathbf{b}\left(\mathbf{v}^{i}\right)\right)\right) .
$$

Since $\mathbb{G}$ is a GLP position auction,

$$
\alpha_{j}\left(v_{i}-p_{j}\left(\mathbf{b}\left(\mathbf{v}^{i}\right)\right)\right)=\alpha_{j}\left(v_{i}-p_{j}(\tilde{\mathbf{b}})\right) .
$$

Hence, we proved (7).

Finally, we show that $\mathbf{m}$ is individually rational. Let $l$ be a player that uses the $T$-strategy and assume player l's value is $v_{l}$. It is enough to show that player $l$ never pays more than $v_{l}$. If all other players choose the mediator, and report $\mathbf{v}_{-l}$, player $l$ 's payment is $p_{l}^{v c g}\left(v_{l}, \mathbf{v}_{-l}\right) \leqslant v_{l}$ since $\varphi^{\mathbf{m}}=\varphi^{v c g}$. Assume not all other players are using the mediator. First consider the case, where $n-1$ players are using the mediator. Let $i \neq l$ be the only player that participates directly in the auction. Let $\mathbf{v}_{-i}$ be the values reported to the mediator by all players but $i$. Let $b_{i}$ be player $i$ 's bid and let $\tilde{\mathbf{b}}=\left(b_{-i}\left(\mathbf{v}^{i}\right), b_{i}\right)$ be the profiles of bids reported to the auctioneer. Let $j=s(\tilde{\mathbf{b}}, i)$ be the position of $i$ resulting from her deviation and let $h=s(\tilde{\mathbf{b}}, l)$ be the position of $l$ resulting from $i$ 's deviation. We need to show that $p_{h}(\tilde{\mathbf{b}}) \leqslant v_{l}$. If $h \in\{m+1,-1\}, p_{h}(\tilde{\mathbf{b}})=0<v_{l}$. Therefore, we can assume without loss of generality that $1 \leqslant h \leqslant m$.

We distinguish between the following cases:

- $h>j$. Observe that $s\left(\mathbf{b}\left(\mathbf{v}^{i}\right), l\right)=s(\tilde{\mathbf{b}}, l)$. Therefore, $s\left(\mathbf{v}^{i}, l\right)=h$. Hence, $\mathbf{v}_{(h)}^{i}=v_{l}$. We now show that $p_{h}(\tilde{\mathbf{b}}) \leqslant v_{l}$. Since $\mathbb{G}$ is a GLP position auction,

$$
p_{h}(\tilde{\mathbf{b}})=p_{h}\left(\mathbf{b}\left(\mathbf{v}^{i}\right)\right) .
$$

By the definition of $\mathbf{b}\left(\mathbf{v}^{i}\right)$,

$$
p_{h}\left(\mathbf{b}\left(\mathbf{v}^{i}\right)\right)=p_{h}^{v c g}\left(\mathbf{v}^{i}\right) .
$$

From the first part of Lemma 3 ,

$$
p_{h}^{v c g}\left(\mathbf{v}^{i}\right) \leqslant v_{(h+1)}^{i} \leqslant v_{(h)}^{i} .
$$

Hence, $p_{h}(\tilde{\mathbf{b}}) \leqslant v_{(h)}^{i}=v_{l}$.

- $h<j$. Observe that $s\left(\mathbf{b}\left(\mathbf{v}^{i}\right), l\right)=h+1$. Therefore, $v_{(h+1)}^{i}=v_{l}$. Since $h<j, \tilde{\mathbf{b}} \leqslant \mathbf{b}\left(\mathbf{v}^{i}\right)$. Therefore, by the monotonicity condition

$$
p_{h}(\tilde{\mathbf{b}}) \leqslant p_{h}\left(\mathbf{b}\left(\mathbf{v}^{i}\right)\right) .
$$

By the definition of $\mathbf{b}\left(\mathbf{v}^{i}\right)$,

$$
p_{h}\left(\mathbf{b}\left(\mathbf{v}^{i}\right)\right)=p_{h}^{v c g}\left(\mathbf{v}^{i}\right) \text {. }
$$

From the first part of Lemma 3

$$
p_{h}^{v c g}\left(\mathbf{v}^{i}\right) \leqslant v_{(h+1)}^{i}=v_{l} .
$$

Therefore

$$
p_{h}(\tilde{\mathbf{b}}) \leqslant v_{l} .
$$


We showed that player $l$, which uses the $T$-strategy does not pay more than her own value, whenever a single player participates directly in the auction. Consider the situation in which a subset of players, which contains more than a single player, participate directly in the auction. The mediator submits the reported values on behalf of the other players. Therefore, by our assumption that players never pay more than their own bid, each of these other players will not pay more than her reported values. In particular, player $l$ will not pay more than her own value. This completes the proof of part (i) of Theorem 4.

The following lemma is needed for the proof of Theorem 5.

Lemma 11. Let $S$ be $s$ strict subset of $\{1,2, \ldots, n\}$. Let $a_{1}, a_{2}, c_{1}, c_{2}$ be real numbers such that $a_{1}<a_{2}$ and $c_{1}<c_{2}$. For every $i=$ $1, \ldots, n$, denote $X_{i}=\left[a_{1}, a_{2}\right]$, and denote $\mathbf{X}=\times_{i=1}^{n} X_{i}$. Let $f: \mathbf{X} \rightarrow\left[c_{1}, c_{2}\right]$ satisfy the following four properties:

(i) $f$ is continuous.

(ii) $f$ is increasing in every $x_{i}$ for every $i \in\{1,2, \ldots, n\} \backslash S$.

(iii) For every $i \in S$ and for every $x_{-i}, f\left(x_{i}, x_{-i}\right)=f\left(y_{i}, x_{-i}\right)$ for every $x_{i}, y_{i} \in X_{i}$.

(iv) $f$ is onto $\left[c_{1}, c_{2}\right]$, that is $f(\mathbf{X})=\left[c_{1}, c_{2}\right]$.

Then, for every $c \in\left(c_{1}, c_{2}\right)$ there exists $\mathbf{x}=\left(x_{1}, x_{2}, \ldots, x_{n}\right) \in X$ with $f(\mathbf{x})=c$ such that $a_{2}>x_{n}>x_{n-1}>\cdots>x_{1}>a_{1}$.

Proof. First note that without loss of generality we can assume that $S=\emptyset$ since we can rename the indices in $\{1,2, \ldots, n\} \backslash S$. Let $c \in\left(c_{1}, c_{2}\right)$. Since $f$ is continuous and increasing in every variable, there exists $0<\epsilon<\frac{a_{2}-a_{1}}{n+1}$ such that $f\left(a_{1}+\epsilon, a_{1}+2 \epsilon, \ldots, a_{1}+n \epsilon\right)<c$ and $f\left(a_{2}-n \epsilon, a_{2}-(n-1) \epsilon, \ldots, a_{2}-\epsilon\right)>c$. Let $g(\delta)=f\left(a_{1}+\epsilon+\delta, a_{1}+2 \epsilon+\delta, \ldots, a_{1}+\right.$ $n \epsilon+\delta)$. Because $g$ is a continuous function of $\delta$ in $\left[0, a_{2}-a_{1}-(n+1) \epsilon\right]$, it obtains any value in $\left[g(0), g\left(a_{2}-a_{1}-(n+1) \epsilon\right)\right]$, and in particular it obtains the value $c$. Let $\delta^{\prime}$ be such that $g\left(\delta^{\prime}\right)=c$. Therefore, $f\left(a_{1}+\epsilon+\delta^{\prime}, a_{1}+2 \epsilon+\delta^{\prime}, \ldots, a_{1}+n \epsilon+\delta^{\prime}\right)=c$, which completes the proof.

Proof of Theorem 5. Let $\mathbb{G}=\mathbb{G}(\alpha, \mathbf{p})$ be a GLP position auction, which satisfies conditions $1-3$. We prove that $\mathbb{G}$ is a VCG cover.

We need the following notation. For every $j \in K$ let $\tilde{D}(j)=\{k: \mathbf{l}(D(j)) \leqslant k<\mathbf{l}(D(j+1))\}$. The following simple claim establishes the non-emptiness of the sets $D(j)$ and $\tilde{D}(j)$ for all $j \in K$, which are used often in the proof.

Claim 1. For every $j \in K:$ (a) $D(j) \neq \emptyset$, (b) $\mathbf{l}(D(j))<\mathbf{l}(D(j+1))$, and (c) $\tilde{D}(j) \neq \emptyset$.

Proof. (a) and (b) follow from condition 1. (c) follows from (a) and (b).

We proceed with the main proof: Let $\mathbf{v} \in V$ be an arbitrary valuation profile. We have to show that there exists $\mathbf{b}(\mathbf{v}) \in \mathbf{B}$ such that $\varphi^{\mathbb{G}}(\mathbf{b}(\mathbf{v}))=\varphi^{v c g}(\mathbf{v})$, where $\varphi^{\mathbb{G}}$ is the outcome function of the position auction $\mathbb{G}$. We begin with recursively constructing a sequence of ordered bid profiles, $\mathbf{z}^{m}, \mathbf{z}^{m-1}, \ldots, \mathbf{z}^{1} \in \mathbf{Z}$, which has the following three properties for every $j \in K$ :

(i) $\tilde{p}_{l}\left(\mathbf{z}^{j}\right)=p_{l}^{v c g}(\mathbf{v})$ for every $l \geqslant j$;

(ii) if $p_{j}^{v c g}(\mathbf{v})>p_{j+1}^{v c g}(\mathbf{v}), \mathbf{z}_{l}^{j}>\mathbf{z}_{l+1}^{j}$ for every $l \in \tilde{D}(j)$;

(iii) $\mathbf{z}_{k}^{j}>0$ for every $k=1, \ldots, n$.

From $\mathbf{z}^{1}$ we will eventually construct $\mathbf{b}(\mathbf{v})$.

Step 1: Constructing $\mathbf{z}^{m}$. We need the following claim:

Claim 2. There exists a vector $\mathbf{z} \in \mathbf{Z}$ such that: (a) $\tilde{p}_{m}(\mathbf{z})=p_{m}^{v c g}(\mathbf{v})$, (b) $z_{k}>0$ for every $k \in \tilde{D}(m)$, and (c) $z_{k}>z_{k+1}$ for every $k \in \tilde{D}(m)$

Proof. First recall that $p_{m}^{v c g}(\mathbf{v})>0$, and that $\tilde{p}_{m+1}(\mathbf{z})=0$ for every $\mathbf{z} \in \mathbf{Z}$. The former holds since $p_{m}^{v c g}(\mathbf{v})=\mathbf{v}_{(m+1)}$ (by the formula of the VCG payment scheme) and $v_{i}>0$ (by assumption). The latter holds because $\tilde{p}_{m+1}(\mathbf{b})=0$ for very bid profile b.

Fix some arbitrary $\mathbf{z} \in \mathbf{Z}$ and let $\hat{c}=p_{m}^{v c g}(\mathbf{v})+1$. Note that $\hat{c} \in\left(\tilde{p}_{m+1}(\mathbf{z}), \infty\right)$. Therefore by condition 1 there exists $\mathbf{z}^{\prime} \in \mathbf{Z}$ such that $\tilde{p}_{m}\left(\mathbf{z}^{\prime}\right)=\hat{c}$. Let $M=z_{1}^{\prime}$ and let $\mathbf{y}$ be defined as follows: $y_{k}=M$ for every $k \leqslant \mathbf{h}(D(m))$, where $\mathbf{h}(D(m))$ denotes the maximal number in $D(m)$, and otherwise $y_{k}=0$. I.e. $\mathbf{y}$ has the following form:

$$
\mathbf{y}=(M, M, \ldots, M, 0,0, \ldots, 0) \text {. }
$$


Obviously $\mathbf{y}$ belongs to $\mathbf{Z}$. In addition, since $M \geqslant z_{k}^{\prime}$ for all $k \in \bigcup_{j \in K} D(j), \tilde{p}_{m}(\mathbf{y}) \geqslant \hat{c}$ by condition 3 . We will next apply Lemma 11.

Let $a=0, b=M, c=0$ and $d=\tilde{p}_{m}(\mathbf{y})$. Let $f:[a, b]^{|\tilde{D}(m)|} \rightarrow[c, d]$ defined by $f(\mathbf{x})=\tilde{p}_{m}\left(y_{-} \tilde{D}(m), \mathbf{x}\right)$. Note that $p_{m}^{v c g}(\mathbf{v}) \in$ $(c, d)$. Hence, by Lemma 11 there exist a vector $\mathbf{x}=\left(x_{1}, \ldots, x_{|\tilde{D}(m)|}\right)$ such that $b>x_{1}>x_{2}>\cdots>x_{|\tilde{D}(m)|}>a$ and $f(\mathbf{x})=$ $p_{m}^{v c g}(\mathbf{v})$.

The vector $\mathbf{z}^{\prime \prime} \in \mathbf{Z}$ defined as follows satisfies (a)-(c): $z_{k+\mathbf{l}(D(m))-1}^{\prime \prime}=x_{k}$ for every $k=1, \ldots,|\tilde{D}(m)|$ and $z_{k}^{\prime \prime}=y_{k}$ otherwise.

We are now ready to define $\mathbf{z}^{m}$. Let $\mathbf{z} \in \mathbf{Z}$ be a vector that satisfies (a)-(c) in Claim 2. Let $z_{k}^{m}=z_{k}$ for every $k \leqslant \mathbf{h}(D(m))$, and let $z_{k}^{m}=\frac{z_{\mathbf{h}(D(m))}}{2}$ for every $k>\mathbf{h}(D(j))$. By construction $\mathbf{z}^{m}$ satisfies (i)-(iii).

Step 2 (The recursive step): Constructing $\mathbf{z}^{j}, j=m-1, \ldots, 1$. Let $j \leqslant m-1$ be fixed and assume that we have already constructed $z^{l}$ for every $l=j+1, \ldots, m$. We construct $\mathbf{z}^{j}$. We distinguish between two cases:

1. $p_{j}^{v c g}(\mathbf{v})=p_{j+1}^{v c g}(\mathbf{v})$ : By condition 1 , since $\tilde{p}_{j+1}\left(\mathbf{z}^{j+1}\right)=p_{j+1}^{v c g}(\mathbf{v})$, there exists a vector $\mathbf{z}^{j} \in Z$, which satisfies: (i) $\tilde{p}_{j}\left(\mathbf{z}^{j}\right)=$ $p_{j}^{v c g}(\mathbf{v})$ and (ii) $z_{k}^{j}>z_{k}^{j+1}$ for every $k \geqslant \mathbf{l}(D(j+1)) . \mathbf{z}^{j} \in Z$ also satisfies (ii) because the conditioning is empty.

2. $p_{j}^{v c g}(\mathbf{v})>p_{j+1}^{v c g}(\mathbf{v})$ : Let $\hat{c}=p_{j}^{v c g}(\mathbf{v})+1$. Hence, $\hat{c} \in\left(\tilde{p}_{j+1}\left(\mathbf{z}^{j+1}\right), \infty\right)$. Therefore, by condition 1 there exists $\mathbf{z}^{\prime} \in \mathbf{Z}$ such that $\tilde{p}_{j}\left(\mathbf{z}^{\prime}\right)=\hat{c}$ and $z_{k}^{\prime}=z_{k}^{j+1}$ for every $k \geqslant \mathbf{l}(D(j+1))$. Let $M=z_{1}^{\prime}$ and let $\mathbf{y}$ be defined as follows: for every $k<\mathbf{l}(D(j+1))$ let $y_{k}=M$, and for all $k: 0 \leqslant k \leqslant n-\mathbf{l}(D(j+1))$ let $y_{\mathbf{l}(D(j+1))+k}=z_{\mathbf{1}(D(j+1))+k}^{j+1}$. That is, $\mathbf{y}$ has the following form:

$$
\mathbf{y}=\left(M, M, \ldots, M, z_{\mathbf{1}(D(j+1))}^{j+1}, z_{\mathbf{l}(D(j+1))+1}^{j+1}, z_{\mathbf{l}(D(j+1))+2}^{j+1}, \ldots\right) .
$$

To obtain $\mathbf{z}^{m}$ follow the analogous arguments as in Step 1 while replacing $m$ with $j$.

Final step: Constructing $\mathbf{b}(\mathbf{v})$ from $\mathbf{z}^{1}$. Define $\mathbf{z}$ to be the following vector: $z_{k}=z_{k}^{1}$ for every $k \geqslant \mathbf{l}(D(1))$, and for every $k<\mathbf{l}(D(1))$, let $z_{k}$ be a distinct coordinate larger than $z_{\mathbf{l}(D(1))}^{1}$, such that $\mathbf{z} \in \mathbf{Z}$. By part (b) of Claim $1 \mathbf{z}$ is well defined.

We are now ready to define $\mathbf{b}=\mathbf{b}(\mathbf{v}) \in B$. For every $i$ such that $s(\mathbf{v}, i) \in K$, let $b_{i}=z_{s(\mathbf{v}, i)}$. Let $S=\{i: s(\mathbf{v}, i) \notin K\}$ and let $T=\left\{z_{m+1}, z_{m+2}, \ldots, z_{n}\right\}$. Note that $|S|=|T|$. For every $i \in S$ we let $b_{i}$ be some element in $T$ such that the following is satisfied: for every pair of players $i, l \in S$, if $i<l$ then $b_{i} \leqslant b_{l}$. By construction we have that $p_{j}(\mathbf{b})=\tilde{p}_{j}(\mathbf{z})=p_{j}^{v c g}(\mathbf{v})$ for every $j \in K$. Therefore, it remains to show $s(\mathbf{b})=s(\mathbf{v})$. Note that by the definition of $\mathbf{b}$, for every pair of players $i, l \in N$, if $s(\mathbf{v}, i)>s(\mathbf{v}, l)$ then $b_{i} \geqslant b_{l}$. Hence, it suffices to show that for every $j \in K$, if $v_{(j)}>v_{(j+1)}$ then $\mathbf{b}_{(j)}>\mathbf{b}_{(j+1)}$. Suppose that $v_{(j)}>v_{(j+1)}$ for some $j \in K$.

We distinguish between the following two cases:

1. $j<\mathbf{l}(D(1))$. By the construction of $\mathbf{z}$ from $\mathbf{z}^{1}$ and the definition of $\mathbf{b}$ we obtain that $\mathbf{b}_{\delta(\mathbf{b}, j)}>\mathbf{b}_{\delta(\mathbf{b}, j+1)}$.

2. $j \geqslant \mathbf{l}(D(1))$. By the second part of Lemma $3, p_{1}^{v c g}(\mathbf{v})>p_{2}^{v c g}(\mathbf{v})>\cdots>p_{j-1}^{v c g}(\mathbf{v})>p_{j}^{v c g}(\mathbf{v})$. Let $\hat{j}=\max \{t: j \geqslant \mathbf{l}(D(t))\}$. Since $\mathbb{G}$ is a GLP position auction, $1<\hat{j}<j$. Since $\mathbf{l}(D(\hat{j}))<\mathbf{l}(D(\hat{j}+1)), \hat{j} \in \tilde{D}(j)$. By the second part of Lemma 3 and since $v_{(j)}>v_{(j+1)}, p_{\hat{j}}^{v c g}(\mathbf{v})>p_{\hat{j}+1}^{v c g}(\mathbf{v})$. Therefore by property (ii) of the sequence we constructed, $z_{j}^{\hat{j}}>z_{j+1}^{\hat{j}}$. Again by construction $z_{j}=z_{j}^{\hat{j}}$ and $z_{j+1}=z_{j+1}^{\hat{j}}$. Therefore, by the definition of $\mathbf{b}$ we obtain that $\mathbf{b}_{(j)}>\mathbf{b}_{(j+1)}$.

We obtained that $\mathbf{b}$ satisfies $\varphi^{\mathbb{G}}(\mathbf{b})=\varphi^{v c g}(\mathbf{v})$, which completes the proof.

Proof of Theorem 6. Let $\mathbb{G}=\mathbb{G}(\alpha, \mathbf{p})$ be a generalized next-price position auction.

We first prove that conditions (i) and (ii) are necessary. Suppose there exists an individually rational mediator that implements $\varphi^{v c g}$ in $\mathbb{G}$. Fix some position $j \in K$. By the second part of Lemma $3, p_{j}^{v c g}(\mathbf{v}) \geqslant p_{j+1}^{v c g}(\mathbf{v})$ for every $\mathbf{v} \in \mathbf{V}$. Therefore, $d(j+1) \geqslant d(j)$ for every $1 \leqslant j \leqslant m-1$. By the second part of Lemma 3 there exists $\mathbf{v}$ for which, $p_{j}^{v c g}(\mathbf{v})>p_{j+1}^{v c g}(\mathbf{v})$, implying that $d(j+1)>d(j)$. This proves that (i) holds. To prove that (ii) holds, note that by the VCG position payment scheme, 3 , for every $\mathbf{v} \in \mathbf{V}$ the player that obtains position $m$ has a positive VCG payment. Therefore, $d(m) \leqslant n$ because otherwise, $p_{m}$ will be identically zero.

We also have to show that conditions (i) and (ii) are sufficient. Indeed, suppose these conditions are satisfied. We have to prove that there exists an individually rational mediator that implements $\varphi^{v c g}$ in $\mathbb{G}$. By Theorem 4 it suffices to prove that $\mathbb{G}$ is a monotone GLP position auction, which is also a VCG cover; the non-trivial task is to show that $\mathbb{G}$ is a VCG cover, and we use Theorem 5 to show it. That is, we have to prove that the three conditions in Theorem 5 are satisfied. Observe that, for every $j \in K, D(j)=\{d(j)\}$. Therefore, only the first condition is not obvious. In order to prove that the first condition in Theorem 5 holds, fix some $\mathbf{z} \in \mathbf{Z}$ and some $c \in\left[\tilde{p}_{j}(\mathbf{b}), \infty\right)$. Let $\mathbf{z}^{\prime}$ be the profile obtained from $\mathbf{z}$ by replacing coordinates $z_{k}$ by $c$ for all $k<d(j+1)$. Since $d(j)<d(j+1), z^{\prime}$ belongs to $\mathbf{Z}$ and $\tilde{p}_{j}\left(\mathbf{z}^{\prime}\right)=c$. 
Proof of Theorem 7. Let $\mathbb{G}=\mathbb{G}(\alpha, \mathbf{p})$ be a weighted next-position auction with weights $c_{1}, c_{2}, \ldots, c_{m}$. We first prove the "only if" part. Let $\hat{j}<m$ be such that $c_{\hat{j}}<c_{\hat{j}+1}$. In order to prove that there does not exist an individually rational mediator that implements $\varphi^{v c g}$ in $\mathbb{G}$ it suffices to show that $\mathbb{G}$ is not a VCG cover. Suppose for contradiction that $\mathbb{G}$ is a VCG cover. Let $\mathbf{v}$ be a vector profile in which $v_{1}=v_{2}=\cdots=v_{n}$. By Lemma $3, p_{\hat{j}}^{v c g}(\mathbf{v})=p_{\hat{j}+1}^{v c g}(\mathbf{v})$. Since $\mathbb{G}$ is a VCG cover there exists $\mathbf{b}$ such that $\psi^{\mathbb{G}}(\mathbf{b})=\varphi^{v c g}(\mathbf{v})$. Therefore,

$$
p_{\hat{j}}(\mathbf{b})=\frac{b_{(\hat{j}+1)}}{c_{\hat{j}}}>\frac{b_{(\hat{j}+2)}}{c_{\hat{j}+1}}=p_{\hat{j}+1}(\mathbf{b}),
$$

contradicting $p_{\hat{j}}(\mathbf{b})=p_{\hat{j}+1}(\mathbf{b})$. We proceed to prove the "if part." Suppose that $c_{1} \geqslant c_{2} \geqslant \cdots \geqslant c_{m}$. We need to prove that there exists an individually rational mediator that implements $\varphi^{v c g}$ in $\mathbb{G}$. By Theorem 4 , it suffices to prove that $\mathbb{G}$ is a monotone GLP position auction, which is also a VCG cover. The only non-obvious task is proving that $\mathbb{G}$ is a VCG cover. However, we omit this proof because it is very similar to the analogous proof of Theorem 6 .

\section{References}

Aghassi, M., Bertsimas, D., 2006. Robust game theory. Math. Programming 107 (1), 231-273.

Ashlagi, I., 2007. A characterization of anonymous truth-revealing position auctions. http://www.people.hbs.edu/iashlagi/papers/TruthfulAnonymous.pdf. Working paper.

Ashlagi, I., Monderer, D., Tennenholtz, M., 2006. Resource selection games with unknown number of players. In: Proceedings of the 5th International Joint Conference on Autonomous Agents and Multiagent Systems, pp. 819-825.

Aumann, R., 1974. Subjectivity and correlation in randomized strategies. J. Math. Econ. 1, 67-96.

Bhat, N., Leyton-Brown, K., Shoham, Y., Tennenholtz, M., 2005. Bidding rings revisited. Working paper.

Borgs, C., Chayes, J.T., Etesami, O., Immorlica, N., Mahdian, M., 2007. Dynamics of bid optimization in online advertisement auctions. In: Proceedings of the 16th International Conference on World Wide Web, pp. 531-540.

Edelman, B., Ostrovsky, M., Schwarz, M., 2007. Internet advertising and the generalized second price auction: Selling billions of dollars worth of keywords. Amer. Econ. Rev. 97.

Feng, J., Bhargava, H., Pennock, D., 2006. Implementing sponsored search in web search engines: Computational evaluation of alternative mechanisms. INFORMS J. Computing.

Forges, F.M., 1986. An approach to communication equilibria. Econometrica 54 (6), 1375-1385.

Graham, D., Marshall, R., 1987. Collusive bidder behavior at single-object second-price and English auctions. J. Polit. Economy 95, 1217-1239.

Holzman, R., Monderer, D., 2004. Characterization of ex post equilibrium in the VCG combinatorial auctions. Games Econ. Behav. 47, 87-103.

Holzman, R., Kfir-Dahav, N., Monderer, D., Tennenholtz, M., 2004. Bundling equilibrium in combinatorial auctions. Games Econ. Behav. 47, $104-123$.

Hyafil, N., Boutilier, C., 2004. Regret minimizing equilibria and mechanisms for games with strict type uncertainty. In: Proceedings of the 20th Annual Conference on Uncertainty in Artificial Intelligence (UAI-04). AUAI Press, Arlington, VA, pp. 268-277.

Kalai, E., Rosenthal, R., 1976. Arbitration of two-party disputes under ignorance. Int. J. Game Theory 7, 65-72.

Lahaie, S., 2006. An analysis of alternative slot auction designs for sponsored search. In: Proceedings of the 7th ACM Conference on Electronic Commerce, pp. 218-227.

Mas-Colell, A., Whinston, W., Green, J., 1995. Microeconomic Theory. Oxford University Press.

McAfee, R., McMillan, J., 1992. Bidding rings. Amer. Econ. Rev. 82, 579-599.

Mehta, A., Saberi, A., Vazirani, V., Vazirani, U., 2007. Adwords and generalized online matching. J. ACM 54 (5).

Monderer, D., Tennenholtz, M., 2004a. K-implementation. J. Artificial Intelligence Res. (JAIR) 21, 37-62.

Monderer, D., Tennenholtz, M., 2004b. K-price auctions: Revenue inequalities, utility equivalence, and competition in auction design. Econ. Theory 24 (2), $255-270$.

Monderer, D., Tennenholtz, M., 2006. Strong mediated equilibrium. In: Proceedings of the AAAI.

Myerson, R.B., 1986. Multistage games with communication. Econometrica 54 (2), 323-358.

Roberts, K., 1979. The characterization of implementable choice rules. In: Laffont, J.-J. (Ed.), Aggregation and Revelation of Preferences. North-Holland Publishing Company.

Rozenfeld, O., Tennenholtz, M., 2007. Routing mediators. In: Proceedings of the 23rd International Joint Conferences on Artificial Intelligence (IJCAI-07), pp. 1488-1493.

Tauman, Y., 2002. A note on k-price auctions with complete information. Games Econ. Behav. 41 (1), 161-164.

Tauman-Kalai, A., Kalai, E., Lehrer, E., Samet, D., 2007. A commitment folk theorem. Working paper. Northwestern University.

Varian, H., 2007. Position auctions. Int. J. Ind. Organ. 25, 1163-1178. 